\title{
Periodic Switched Control of Dual-Rate Sampled-Data Systems
}

\author{
Dawei Zhang, Wei Bai, and Xinchun Jia \\ School of Mathematical Sciences, Shanxi University, Taiyuan 030006, China \\ Correspondence should be addressed to Dawei Zhang; zhangdaweisx@sxu.edu.cn
}

Received 21 October 2014; Revised 9 April 2015; Accepted 15 April 2015

Academic Editor: Dan Simon

Copyright (C) 2015 Dawei Zhang et al. This is an open access article distributed under the Creative Commons Attribution License, which permits unrestricted use, distribution, and reproduction in any medium, provided the original work is properly cited.

\begin{abstract}
This paper is concerned with the periodic switched control of a linear dual-rate sampled-data system. The state variables of the continuous-time plant are sampled by two types of sensors. The ratio of two sampling rates is assumed to be a rational number. Depending on whether the sampled-data of state variables at two sampling rates is available simultaneously or separately, a periodic switched controller is constructed. Applying an input delay approach, the closed-loop system is modeled as a switched system with subsystems having different input delays. Some delay-dependent criteria for the $H_{\infty}$ performance of the switched system and the existence of the switched controller are derived by employing a Lyapunov-Krasovskii functional that includes information about two sampling periods. The dual-rate sampled-data control of a vehicle dynamic system is given to show that the proposed method is effective and it can achieve a better $H_{\infty}$ control performance than the single-rate design method.
\end{abstract}

\section{Introduction}

Sampled-data control of continuous-time practical systems, especially complex industrial systems, offers several advantages such as flexibility, low cost, and increased reliability $[1,2]$. In a sampled-data control system, where a continuoustime plant is controlled with a digital controller, the sampling rate is a critical design parameter. The choice of the sampling rate mainly depends on some factors like bandwidth and response time of closed-loop systems, physical limitations of sensors and actuators, and the effect of the noise. The sampling rate must be chosen as fast as possible to ensure the high precision and the fast response time of the system but as slow as possible to satisfy the hardware limits and to eliminate the effect of the noise on the control input. Taking these factors into account, the effective range of the sampling rate is determined.

In many industrial applications, it is impractical to sample all physical signals uniformly at one single-rate, which demands a multirate sampling scheme. For instance, for an industrial vehicle, laser sensors are used to measure the heave position and the heave velocity, and gyrometers are chosen to measure the angular velocity and the heading angle [3]. Due to the sensor restrictions and the control performance requirement, it is often necessary to sample the signals for different types of sensors at different sampling rates. The multirate sampling technique has received much attention since the early 1950s. Compared with the singlerate sampling scheme, the use of the multirate sampling technique is of two main benefits: (i) it may improve the performance such as improving the transient system behavior and enhancing the disturbance rejection property and (ii) it can provide a better tradeoff between the system performance and the implementation cost, which can be achieved by using analog-to-digital converters and digital-to-analog converters at different rates. Motivated by these benefits, much work has been done to deal with system modeling and identification, stability analysis, and controller synthesis of multirate sampled-data systems in the past few decades [3-12]. For example, in [4], a general framework of a multirate sampleddata control system is presented using nest operators and nest algebras, and an $H_{\infty}$ suboptimal controller satisfying causality constraint is designed by the lifting technique, in which the outputs $y_{i}(t)(i=1,2, \ldots, p)$ and the control inputs $u_{i}(t)(i=1,2, \ldots, q)$ are paired with sampling periods $h_{s}=m_{i} h$ and holding periods $h_{u}=n_{i} h$, respectively, where $m_{i} \in \mathbb{N}^{+}(i=1,2, \ldots, p), n_{i} \in \mathbb{N}^{+}(i=1,2, \ldots, q), \mathbb{N}^{+}$ is the set of positive integers, and $h$ is the base sampling period. Based on this framework, some particular cases of the multirate sampled-data systems are considered in [5-15]. 
More specifically, when the multirate sampled-data system involves a fast sampling rate $1 / h_{s}$ and a slow control input rate $1 / h_{u}$ (i.e., $n / h_{u}=1 / h_{s}\left(n \in \mathbb{N}^{+}\right)$), a new multirate sampling method for acceleration control is proposed in [9]; on the other hand, when the multirate sampled-data system involves a slow sampling rate $1 / h_{s}$ and a fast control input rate $1 / h_{u}$ (i.e., $n / h_{s}=1 / h_{u}$ ), some estimation and/or control problems for several practical systems such as polymer reactors [10], visual servo control systems [11, 12], read-write arm of the hard disk drive [13, 14], and a pilot plant [15] are addressed. It should be mentioned that for the multirate sampling scheme with $h_{u}>h_{s}$, in [9], the control signal is calculated by output measurement at each sampling rate $1 / h_{s}$, but only the one produced at the input rate $1 / h_{u}$ is implemented. Although such a multirate scheme is effective in the realization of acceleration control in wide bandwidth, some of the output measurements may not be used to update the control actuation in time; for the multirate sampling scheme with $h_{u}<h_{s}$ in $[3,5-8,10-15]$, the control signal is calculated recursively at each input rate $1 / h_{u}$ only using the available data at each sampling rate $1 / h_{s}$. Moreover, using the lifting technique, the multirate sampled-data system is converted into an equivalent discrete-time single-rate timeinvariant system in $[3,5-8,10-15]$. However, the equivalent conversion is not readily applicable to the continuous-time systems with polytopic uncertainties [16-18]. In [16], an input delay approach is proposed to investigate the sampled-data stabilization of linear systems, which can be extended to deal with the sampled-data control for systems with polytopic uncertainties and the networked control systems [19-24]. Most of the existing results developed by using the input delay approach, such as [16-18], have been largely focused on the sampled-data control of single-rate sampled-data systems. However, there are few results available on the dualrate or multirate sampled-data control of a continuous-time system using the input delay approach except [20,25], which provides the main motivation of the current study. In [20], exponential stability and the induced $L_{2}$-gain of networked control systems are investigated, in which the sampled-data via dual-rate samplings are transmitted one after another by introducing a Round-Robin scheduling protocol. In [25], multirate sampled-data systems are modeled as systems with multiple input delays by reordering the updating instants, and some stability and stabilization conditions are established in terms of linear matrix inequalities. Without reordering sensor instants [20] or updating instants [25], this paper attempts to apply the input delay approach for a dual-rate sampled-data control system.

In this paper, we apply the input delay approach to investigate the periodic switched control of a linear continuoustime system system with two different sampling rates $1 / h_{1}$ and $1 / h_{2}$, where the sampling periods $h_{1}$ and $h_{2}$ satisfy $h_{1}<h_{2}$ and $l_{1} h_{1}=l_{2} h_{2}$, with $l_{i}(i=1,2)$ being two positive integers, $l_{i} h_{i}$ being the unique basic time period, and $\left\{l_{1}, l_{2}\right\}$ having no common factors greater than unity. Once the sampled-data of the state variable $x_{1}\left(k h_{1}\right)$ or $x_{2}\left(k h_{2}\right)$ is available, the control input is computed to update the system. Depending on whether the sampled-data of state variables at two sampling rates are available simultaneously or separately, a periodic switched controller with three switching modes is constructed to implement the sampled-data control. Using such a controller and the input delay approach, the resulting closed-loop system is modeled as a switched system with subsystems that have different input delays. A LyapunovKrasovskii functional that involves information about two sampling periods is constructed to derive some delaydependent criteria for the $H_{\infty}$ performance of the switched system and the existence of the periodic switched controller. Comparing with the existing results for multirate sampleddata systems based on lifting technique [3-15], the proposed results can be trivially extended to handle the multirate sampled-data systems or networked control systems with polytopic uncertainties. The effectiveness of the proposed method and its advantage over a single-rate sampled-data control is shown by performing the dual-rate sampled-data control of a vehicle system.

Notation. The superscript " $T$ " stands for the transposition of a vector or a matrix. $\mathbb{R}^{n}$ is the $n$ dimensional Euclidean space. $\mathbb{N}$ is the set of nonnegative integers and $\mathbb{N}^{+}$is the set of positive integers. For symmetric matrices $P$ and $Q, P \leq Q$ (resp., $P<$ $Q)$ means that $P-Q$ is negative semidefinite matrix (resp., negative definite matrix). $\lambda_{\max }(P)$ is the maximum eigenvalue of a symmetric matrix $P$. We use an asterisk "*” to denote a term induced by symmetry and $\operatorname{diag}\{\cdots\}$ to denote the block-diagonal matrix. The space of square-integrable vector functions over $\left[t_{0}, \infty\right)$ is denoted by $\mathscr{L}_{2}\left[t_{0}, \infty\right)$.

\section{Modeling of a Dual-Rate Sampled-Data System with a Switched Controller}

Consider the linear system described by

$$
\begin{aligned}
& \dot{x}(t)=A x(t)+B u(t)+E \omega(t), \\
& z(t)=C x(t)+D \omega(t),
\end{aligned}
$$

where $x(t) \in \mathbb{R}^{n}, u(t) \in \mathbb{R}^{m}$, and $z(t) \in \mathbb{R}^{p}$ are the state, the control input, and the controlled output, respectively; $\omega(t) \in \mathbb{R}^{q}$ is the external disturbance acting on system (1) and $\omega(t) \in \mathscr{L}_{2}\left[t_{0}, \infty\right) ; x\left(t_{0}\right)=x_{0}$ is the initial state; $A, B, C$, $D$, and $E$ are constant matrices of appropriate dimensions. It is assumed that all state variables of system (1) are sampled by two different types of sensors. Let $x(t)=\left[\begin{array}{ll}x_{1}^{T}(t) & x_{2}^{T}(t)\end{array}\right]^{T}$, where $x_{1}(t)=\left[\begin{array}{llll}x_{1,1}^{T}(t) & x_{1,2}^{T}(t) & \cdots & x_{1, p_{1}}^{T}(t)\end{array}\right]^{T} \in \mathbb{R}^{p_{1}}, x_{2}(t)=$ $\left[\begin{array}{llll}x_{2,1}^{T}(t) & x_{2,2}^{T}(t) & \cdots & x_{2, p_{2}}^{T}(t)\end{array}\right]^{T} \in \mathbb{R}^{p_{2}}$, and $p_{1}+p_{2}=n$. Without loss of generality, we assume that $x_{1}(t)$ and $x_{2}(t)$ are paired with two sampling periods $h_{1}$ and $h_{2}$, respectively, and $l_{1} h_{1}=l_{2} h_{2}\left(h_{1} \leq h_{2}\right)$, where $l_{i} \in \mathbb{N}^{+}(i=1,2)$ and $l_{1} \geq l_{2}$. Then the sequences of sampled-data of the state variables $x_{1}(t)$ and $x_{2}(t)$ are $\left\{x_{1}\left(k h_{1}\right): k \in \mathbb{N}\right\}$ and $\left\{x_{2}\left(k h_{2}\right): k \in \mathbb{N}\right\}$.

In the proposed dual-rate sampling scheme, once $x_{1}\left(k h_{1}\right)$ or $x_{2}\left(k h_{2}\right)$ is available, the control signal is computed immediately for input update. By taking full advantage of $x_{1}\left(k h_{1}\right)$ and $x_{2}\left(k h_{2}\right)(k \in \mathbb{N})$ in a real-time way, we construct the following switched controller, which is shown in Figure 1:

$$
u\left(t^{+}\right)=F_{1, \sigma(t)} x_{1}\left(k h_{1}\right)+F_{2, \sigma(t)} x_{2}\left(k h_{2}\right), \quad \forall k \in \mathbb{N},
$$



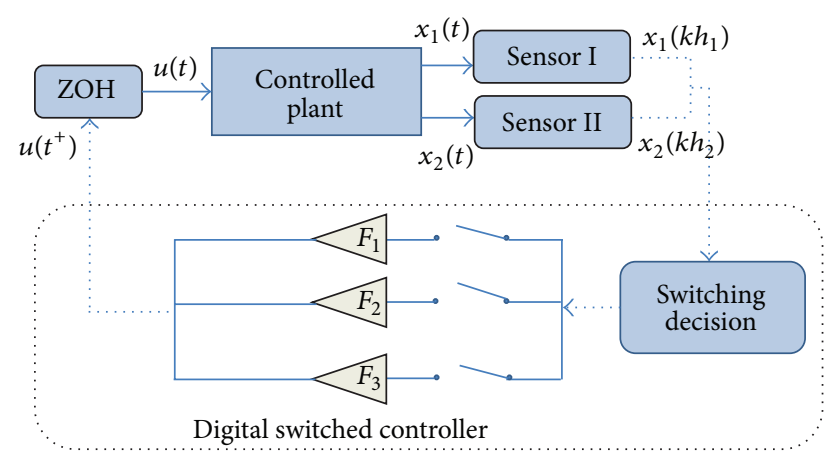

Figure 1: The multirate sampled-data system architecture using a digital switched controller.

where $F_{\sigma(t)}=\left[\begin{array}{ll}F_{1 \sigma(t)} & F_{2 \sigma(t)}\end{array}\right]$ are the control gain matrices to be determined and $\sigma(t):[0, \infty) \rightarrow \mathbb{S}=\{1,2,3\}$ is the switching signal. Notice that there are three cases of available sampled-data of the state variables for control computation of the controller (2): (i) both $x_{1}\left(k h_{1}\right)$ and $x_{2}\left(k h_{2}\right)$ are available, (ii) only $x_{1}\left(k h_{1}\right)$ is available, and (iii) only $x_{2}\left(k h_{2}\right)$ is available. Define the switching rules as follows. Set $\sigma(t)=1$ when $x_{1}\left(k h_{1}\right)$ and $x_{2}\left(k h_{2}\right)$ are available simultaneously, $\sigma(t)=2$ when only $x_{1}\left(k h_{1}\right)$ is available, and $\sigma(t)=3$ when only $x_{2}\left(k h_{2}\right)$ is available, respectively.

Due to the fact that $l_{1} h_{1}=l_{2} h_{2}$, the interval of the control input is $\left[k l_{1} h_{1},(k+1) l_{1} h_{1}\right)$ or equally $\left[k l_{2} h_{2},(k+1) l_{2} h_{2}\right)(k \in$ $\mathbb{N})$. Define $m_{i}=\max \left\{k \mid k h_{1}-i h_{2}<0,0<k<l_{1}, k \in\right.$
$\mathbb{N}\}\left(i=1,2, \ldots, l_{2}\right)$ and $m_{0}=0$. Based on the switching rules of controller (2), we propose the following interval partition:

$$
\begin{aligned}
{\left[k l_{1} h_{1},(k+1) l_{1} h_{1}\right)=} & \mathscr{I}_{1} \cup \mathscr{I}_{2} \cup \cdots \cup \mathscr{I}_{m_{\nu-1}+v} \\
& \cup \mathscr{I}_{m_{\nu-1}+v+1} \cup \cdots \cup \mathscr{I}_{m_{\nu}+\nu} \cup \cdots \\
& \cup \mathscr{I}_{m_{l_{2}+1}+l_{2}} \cup \cdots \cup \mathscr{I}_{m_{l_{2}}+l_{2}},
\end{aligned}
$$

where

$$
\begin{aligned}
& \mathscr{I}_{m_{\nu-1}+v}=\left[k l_{1} h_{1}+(\nu-1) h_{2},\left(k l_{1}+m_{\nu-1}+1\right) h_{1}\right), \\
& \mathscr{I}_{m_{\nu-1}+\nu+1} \\
& \quad=\left[\left(k l_{1}+m_{\nu-1}+1\right) h_{1},\left(k l_{1}+m_{\nu-1}+2\right) h_{1}\right), \\
& \vdots \\
& \mathscr{I}_{m_{\nu}+\nu}=\left[\left(k l_{1}+m_{\nu}\right) h_{1}, k l_{1} h_{1}+v h_{2}\right), \\
& \quad v=1,2, \ldots, l_{2} .
\end{aligned}
$$

To clearly show the partition process, a special case for the interval partition of $\left[k l_{1} h_{1},(k+1) l_{1} h_{1}\right)$ with $l_{1}=8$ and $l_{2}=3$ is depicted by Figure 2 .

Considering interval partition (3) and the availability of $x_{1}\left(k h_{1}\right)$ and $x_{2}\left(k h_{2}\right)$, one can see that switching controller (2) is periodically activated on the time instants $\left\{k l_{1} h_{1}+\left(m_{i-1}+\right.\right.$ 1) $\left.h_{1}, k l_{1} h_{1}+(i-1) h_{2}, i=1, \ldots, l_{2}\right\}_{k=0}^{\infty}$. Then the control input can be described by

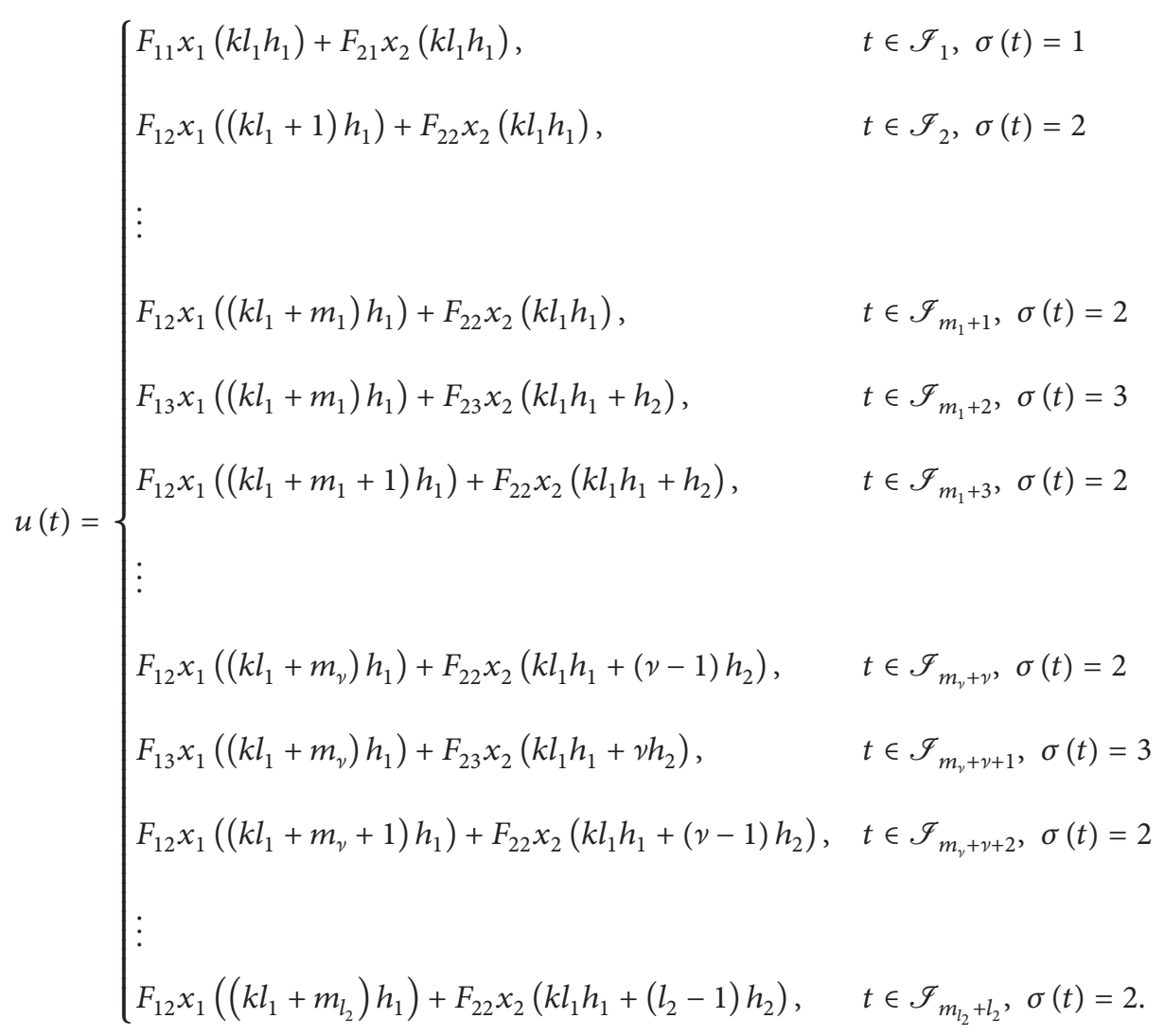




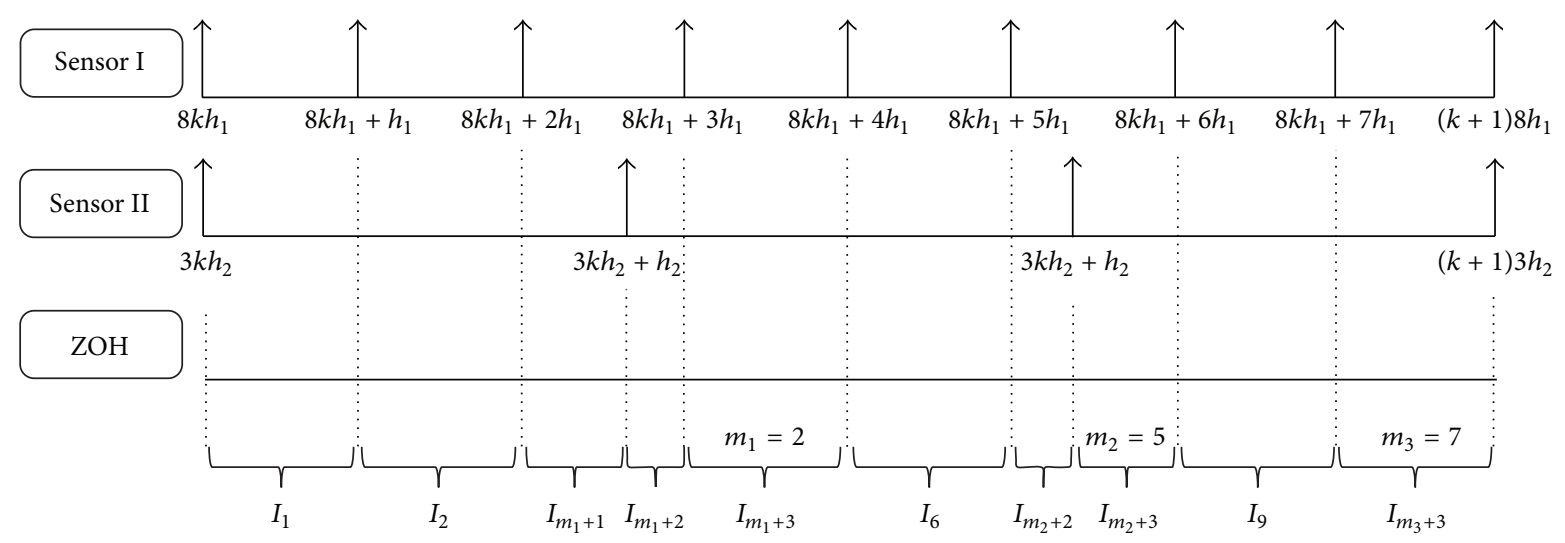

FIGURE 2: A special case for the interval partition of $\left[k l_{1} h_{1},(k+1) l_{1} h_{1}\right)$ with $l_{1}=8$ and $l_{2}=3$.

Define the following input delays on the subintervals in (3):

$$
\begin{aligned}
& t \in \mathscr{I}_{m_{v-1}+\nu}: \\
& \tau_{m_{\nu-1}+v}^{(1)}(t)=t-\left(k l_{1}+m_{\nu-1}\right) h_{1}, \\
& \tau_{m_{\nu-1}+v}^{(2)}(t)=t-k l_{1} h_{1}-(\nu-1) h_{2} \\
& t \in \mathscr{I}_{m_{\nu-1}+\nu+1}: \\
& \tau_{m_{\nu-1}+v+1}^{(1)}(t)=t-\left(k l_{1}+m_{\nu-1}+1\right) h_{1} \text {, } \\
& \tau_{m_{\nu-1}+\nu+1}^{(2)}(t)=t-k l_{1} h_{1}-(\nu-1) h_{2} \\
& \sigma(t)=1: \quad 0 \leq \tau_{1}(t)<h_{1}, t \in \mathscr{I}_{1} \\
& \sigma(t)=2: \\
& 0 \leq \tau_{i}^{(1)}(t)<h_{1}, \quad 0 \leq \tau_{i}^{(1)}(t)<\tau_{i}^{(2)}(t)<h_{2}, t \in \mathscr{I}_{i}, i=m_{v-1}+v+1, \ldots, m_{v}+v, v=1,2, \ldots, l_{2} \\
& \sigma(t)=3: \quad 0 \leq \tau_{m_{v-1}+v}^{(2)}(t)<\tau_{m_{v-1}+v}^{(1)}(t)<h_{1}, t \in \mathscr{I}_{m_{v-1}+v}, \quad v=2, \ldots, l_{2} \text {. } \\
& t \in \mathscr{I}_{m_{v}+v}: \\
& \tau_{m_{\nu}+v}^{(1)}(t)=t-\left(k l_{1}+m_{\nu}\right) h_{1} \\
& \tau_{m_{v}+v}^{(2)}(t)=t-k l_{1} h_{1}-(\nu-1) h_{2}
\end{aligned}
$$

Using (1), (5), and (6), the resulting closed-loop system can be described by

$$
\begin{aligned}
& \Sigma_{1}^{(1)}: \dot{x}(t)=A x(t)+B F_{1} x\left(t-\tau_{1}(t)\right)+E \omega(t), \quad t \in \mathscr{I}_{1}, \sigma(t)=1, \\
& \Sigma_{2 v}^{(2)}: \dot{x}(t)=A x(t)+B F_{2} I_{p_{1}} x\left(t-\tau_{i}^{(1)}(t)\right)+B F_{2} I_{p_{2}} x\left(t-\tau_{i}^{(2)}(t)\right)+E \omega(t), \\
& t \in \bigcup_{m_{\nu-1}+v+1}^{m_{\nu}+v} \mathscr{I}_{i}, i=m_{\nu-1}+v+1, \ldots, m_{v}+v, v=1,2, \ldots, l_{2}, \sigma(t)=2 \text {, } \\
& \Sigma_{2 \nu-1}^{(3)}: \dot{x}(t)=A x(t)+B F_{3} I_{p_{1}} x\left(t-\tau_{m_{\nu-1}+v}^{(1)}(t)\right)+B F_{3} I_{p_{2}} x\left(t-\tau_{m_{\nu-1}+v}^{(2)}(t)\right)+E \omega(t), \\
& t \in \mathscr{I}_{m_{v-1}+v}, v=2, \ldots, l_{2}, \sigma(t)=3,
\end{aligned}
$$


where $I_{p_{1}}=\operatorname{diag}\left\{I_{p_{1} \times p_{1}}, 0\right\}, I_{p_{2}}=\operatorname{diag}\left\{0, I_{p_{2} \times p_{2}}\right\}$ and $I_{p_{1} \times p_{1}}$ and $I_{p_{2} \times p_{2}}$ denote $p_{1} \times p_{1}$ and $p_{2} \times p_{2}$ identity matrices, respectively.

The purpose of this paper is to investigate the periodic switched control such that system (8) is exponentially stable with a prescribed $H_{\infty}$ performance, which means that

(i) system (8) with $\omega(t)=0$ is exponentially stable; that is, there exist constants $\beta>0$ and $\lambda>0$ such that $\|x(t)\|^{2} \leq \beta\left\|x_{0}\right\|_{c 1} e^{-\lambda t}$ for $t \geq 0$, where $\left\|x_{t}\right\|_{c 1}=$ $\sup _{-h_{2} \leq s \leq 0}\{\|x(t+s)\|,\|\dot{x}(t+s)\|\}[23,26] ;$

(ii) $\int_{0}^{\infty} e^{-\alpha s} z^{T}(s) z(s) d s \leq \gamma^{2} \int_{0}^{\infty} \omega^{T}(s) \omega(s) d s$ can be ensured for all nonzero $\omega(t) \in \mathscr{L}_{2}[0, \infty)$ under the zero initial condition $x(t)=\phi(t)=0\left(t \in\left[-h_{2}, 0\right]\right)$, where $\alpha$ and $\gamma$ are positive constants.

To end this section, we introduce the following lemma.

Lemma 1. For any constant matrices $R \in \mathbb{R}^{n \times n}, S_{i} \in \mathbb{R}^{n \times n}$ (i $=1,2), \mathfrak{R}_{1}=\left[\begin{array}{ll}R & S_{1}^{T} \\ S_{1} & R\end{array}\right] \geq 0, \mathfrak{R}_{2}=\left[\begin{array}{ll}R & S_{2}^{T} \\ S_{2} & R\end{array}\right] \geq 0$, and scalars $\tau_{i}, \tau_{i}(t)(i=1,2)$ satisfying $0 \leq \tau_{1} \leq \tau_{1}(t)<\tau_{2}(t) \leq \tau_{2}$ and $a$ vector function $\dot{x}:\left[-\tau_{2},-\tau_{1}\right] \rightarrow \mathbb{R}^{n}$ such that the integration concerned is well defined, the following inequality holds:

$$
-\left(\tau_{2}-\tau_{1}\right) \int_{t-\tau_{2}}^{t-\tau_{1}} \dot{x}^{T}(s) R \dot{x}(s) d s \leq-\sum_{i=1}^{7} \mathscr{E}_{i},
$$

where

$$
\begin{aligned}
\mathscr{E}_{1} & =e_{3}^{T}(t) \operatorname{Re}_{3}(t), \\
\mathscr{E}_{2} & =e_{2}^{T}(t) \operatorname{Re}_{2}(t), \\
\mathscr{E}_{3} & =e_{1}^{T}(t) \operatorname{Re}_{1}(t), \\
\mathscr{E}_{4} & =e_{1}^{T}(t) S_{1}^{T} e_{2}(t), \\
\mathscr{E}_{5} & =e_{2}^{T}(t) S_{1} e_{1}(t), \\
\mathscr{E}_{6} & =e_{2}^{T}(t) S_{2}^{T} e_{3}(t), \\
\mathscr{E}_{7} & =e_{3}^{T}(t) S_{2} e_{2}(t), \\
e_{1}(t) & =x\left(t-\tau_{1}\right)-x\left(t-\tau_{1}(t)\right), \\
e_{2}(t) & =x\left(t-\tau_{1}(t)\right)-x\left(t-\tau_{2}(t)\right), \\
e_{3}(t) & =x\left(t-\tau_{2}(t)\right)-x\left(t-\tau_{2}\right) .
\end{aligned}
$$

Proof. If $\tau_{1}(t) \equiv \tau_{1}$ (resp., $\tau_{2}(t) \equiv \tau_{2}$ ), inequality (9) reduces to be the one in Lemma 1 [27]. If $0 \leq \tau_{1} \leq \tau_{1}(t)<\tau_{2}(t) \leq \tau_{2}$, using Jensen inequality to obtain

$$
\begin{aligned}
-\left(\tau_{2}-\tau_{1}\right) \int_{t-\tau_{2}}^{t-\tau_{1}} \dot{x}^{T}(s) R \dot{x}(s) d s \\
\leq- \\
\leq\left(1+\frac{\alpha_{1}}{\alpha_{2}}\right) \mathscr{E}_{1}-\left(1+\frac{\alpha_{2}}{\alpha_{1}}+\frac{\alpha_{4}}{\alpha_{3}}\right) \mathscr{E}_{2} \\
-\left(1+\frac{\alpha_{3}}{\alpha_{4}}\right) \mathscr{E}_{3},
\end{aligned}
$$

where

$$
\begin{aligned}
& \alpha_{1}=\frac{\tau_{2}(t)-\tau_{1}}{\tau_{2}-\tau_{1}}, \\
& \alpha_{2}=\frac{\tau_{2}-\tau_{2}(t)}{\tau_{2}-\tau_{1}}, \\
& \alpha_{3}=\frac{\tau_{2}-\tau_{1}(t)}{\tau_{2}-\tau_{1}}, \\
& \alpha_{4}=\frac{\tau_{1}(t)-\tau_{1}}{\tau_{2}-\tau_{1}},
\end{aligned}
$$

for any $\mathfrak{R}_{i} \geq 0(i=1,2)$, the following inequalities hold:

$$
\begin{aligned}
& {\left[\begin{array}{c}
\sqrt{\frac{\alpha_{3}}{\alpha_{4}}} e_{1}(t) \\
-\sqrt{\frac{\alpha_{4}}{\alpha_{3}}} e_{2}(t)
\end{array}\right]^{T} \Re_{1}\left[\begin{array}{c}
\sqrt{\frac{\alpha_{3}}{\alpha_{4}}} e_{1}(t) \\
-\sqrt{\frac{\alpha_{4}}{\alpha_{3}}} e_{2}(t)
\end{array}\right] \geq 0,} \\
& {\left[\begin{array}{c}
\sqrt{\frac{\alpha_{2}}{\alpha_{1}}} e_{2}(t) \\
-\sqrt{\frac{\alpha_{1}}{\alpha_{2}}} e_{3}(t)
\end{array}\right]^{T} \mathfrak{R}_{2}\left[\begin{array}{c}
\sqrt{\frac{\alpha_{2}}{\alpha_{1}}} e_{2}(t) \\
-\sqrt{\frac{\alpha_{1}}{\alpha_{2}}} e_{3}(t)
\end{array}\right] \geq 0 .}
\end{aligned}
$$

It follows from (13) that

$$
\begin{aligned}
& \frac{\alpha_{3}}{\alpha_{4}} \mathscr{E}_{3}+\frac{\alpha_{4}}{\alpha_{3}} \mathscr{E}_{2} \geq \mathscr{E}_{4}+\mathscr{E}_{5}, \\
& \frac{\alpha_{2}}{\alpha_{1}} \mathscr{E}_{2}+\frac{\alpha_{1}}{\alpha_{2}} \mathscr{E}_{1} \geq \mathscr{E}_{6}+\mathscr{E}_{7} .
\end{aligned}
$$

Combining (14) and (15), we obtain inequality (9). This completes the proof.

\section{3. $H_{\infty}$ Performance Analysis and Periodic Switched Controller Design}

In this section, we first derive a delay-dependent criterion such that system (8) is exponentially stable with prescribed $H_{\infty}$ performance by using the Lyapunov-Krasovskii functional method. Based on the derived performance criterion, we establish the delay-dependent criterion on the existence of a switched controller for system (8).

Proposition 2. Given $\gamma>0, h_{2}>h_{1}>0, l_{1}>l_{2}>0,0 \leq$ $a_{1} \leq a_{2} \leq a_{3} \leq\left(\left(l_{2}-2\right) a_{1}+\left(l_{2}+3\right) a_{2}\right) /\left(l_{2}+1\right), \mu \geq 1$ satisfying $\ln (\mu)<\left[\left(a_{1}\left(l_{2}-2\right)+a_{2}\left(l_{2}+3\right)-a_{3}\left(l_{2}+1\right)\right) / l_{2}\right]\left(h_{2} / 2\right)$, and the gain matrices $F_{i}(i=1,2,3)$, system $(8)$ is exponentially stable with a given $H_{\infty}$ performance $\gamma$ for any switching signal with $\ln (\mu)<\left[\left(a_{1}\left(l_{2}-2\right)+a_{2}\left(l_{2}+3\right)-a_{3}\left(l_{2}+1\right)\right) / l_{2}\right]\left(h_{2} / 2\right)$, if there exist matrices $S_{1}, S_{2}, S_{i, 1}$, and $S_{i, 2}(i=2,3)$ and symmetric matrices $P_{j}>0, Q_{i j}>0$, and $R_{i j}>0(i=1,2 ; j=1,2,3)$ such that $P_{2} \leq \mu P_{1}, P_{1} \leq \mu P_{2}, P_{2} \leq \mu P_{3}, P_{3} \leq \mu P_{2}, Q_{i 2} \leq \mu Q_{i 1}$, 
$Q_{i 1} \leq \mu Q_{i 2}, Q_{i 2} \leq \mu Q_{i 3}, Q_{i 3} \leq \mu Q_{i 2}, R_{i 2} \leq \mu R_{i 1}, R_{i 1} \leq \mu R_{i 2}$, $R_{i 2} \leq \mu R_{i 3}, R_{i 3} \leq \mu R_{i 2}(i=1,2)$, and

$$
\begin{gathered}
{\left[\begin{array}{cc}
R_{11} & S_{1}^{T} \\
S_{1} & R_{11}
\end{array}\right] \geq 0,} \\
{\left[\begin{array}{cc}
h_{1} R_{12}-h_{12} R_{22} & S_{2}^{T} \\
S_{2} & h_{1} R_{12}-h_{12} R_{22}
\end{array}\right] \geq 0,} \\
{\left[\begin{array}{ll}
R_{1 i} & S_{i, 1}^{T} \\
S_{i, 1} & R_{1 i}
\end{array}\right] \geq 0, \quad(i=2,3)} \\
{\left[\begin{array}{cc}
R_{1 i} & S_{i, 2}^{T} \\
S_{i, 2} & R_{1 i}
\end{array}\right] \geq 0, \quad(i=2,3)} \\
\Xi^{(1)}<0, \quad t \in \mathscr{I}_{1}, \\
\Xi^{(i 1)}<0, \\
t \in \mathscr{I}_{i}, i=m_{v-1}+v+1, \ldots, m_{v}+v, v=1,2, \ldots, l_{2}, \\
\Xi^{(i 2)}<0,
\end{gathered}
$$

where

$$
\begin{aligned}
\Xi^{(1)}= & {\left[\begin{array}{ccccc}
\Xi_{11}^{(1)} & \Xi_{12}^{(1)} & \Xi_{13}^{(1)} & 0 & P_{1} E+C^{T} D \\
* & \Xi_{22}^{(1)} & \Xi_{23}^{(1)} & 0 & 0 \\
* & * & \Xi_{33}^{(1)} & \Xi_{34}^{(1)} & 0 \\
* & * & * & \Xi_{44}^{(1)} & 0 \\
* & * & * & * & -\gamma^{2} I+D^{T} D
\end{array}\right] } \\
& +\Psi_{1}^{T}\left(h_{1}^{2} R_{11}+h_{12}^{2} R_{21}\right) \Psi_{1}, \\
\Xi_{11}^{(1)}= & A^{T} P_{1}+P_{1} A+a_{1} P_{1}+Q_{11}-\sigma_{11} R_{11}+C^{T} C, \\
\Xi_{12}^{(1)}= & P_{1} B F_{1}+\sigma_{11}\left(R_{11}-S_{1}^{T}\right), \\
\Xi_{13}^{(1)}= & \sigma_{11} S_{1}^{T}, \\
\Xi_{22}^{(1)}= & -\sigma_{11}\left(2 R_{11}-S_{1}^{T}-S_{1}\right), \\
\Xi_{23}^{(1)}= & \sigma_{11}\left(R_{11}-S_{1}^{T}\right), \\
\Xi_{33}^{(1)}= & -\sigma_{11} R_{11}-\sigma_{21} R_{21}+\sigma_{11}\left(Q_{21}-Q_{11}\right), \\
\Xi_{34}^{(1)}= & \sigma_{21} R_{21}, \\
\Xi_{44}^{(1)}= & -\sigma_{21}\left(R_{21}+Q_{21}\right),
\end{aligned}
$$

$$
\begin{aligned}
& \Psi_{1}=\left[\begin{array}{lllll}
A & B F_{1} & 0 & 0 & E
\end{array}\right], \\
& \sigma_{11}=e^{-a_{1} h_{1}} \text {, } \\
& \sigma_{21}=e^{-a_{1} h_{2}} \\
& \Xi^{(i 1)}=\left[\begin{array}{cccccc}
\Xi_{11}^{(i 1)} & \Xi_{12}^{(i 1)} & \Xi_{13}^{(i 1)} & \Xi_{14}^{(i 1)} & 0 & \Xi_{16}^{(i 1)} \\
* & \Xi_{22}^{(i 1)} & \Xi_{23}^{(i 1)} & \Xi_{24}^{(i 1)} & \Xi_{25}^{(i 1)} & 0 \\
* & * & \Xi_{33}^{(i 1)} & 0 & \Xi_{35}^{(i 1)} & 0 \\
* & * & * & \Xi_{44}^{(i 1)} & 0 & 0 \\
* & * & * & * & \Xi_{55}^{(i 1)} & 0 \\
* & * & * & * & * & \Xi_{66}^{(i 1)}
\end{array}\right] \\
& +\Psi_{2}^{T}\left(h_{1}^{2} R_{12}+h_{12}^{2} R_{22}\right) \Psi_{2} \\
& \Xi_{11}^{(i 1)}=A^{T} P_{2}+P_{2} A+a_{2} P_{2}+Q_{12}+C^{\mathrm{T}} C-\sigma_{22} R_{22} \\
& -\sigma_{12}\left(h_{1} R_{12}-h_{12} R_{22}\right) \text {, } \\
& \Xi_{12}^{(i 1)}=P_{2} B F_{12}+\sigma_{12}\left(h_{1} R_{12}-h_{12} R_{22}-S_{2}^{T}\right) \\
& +\sigma_{22}\left(R_{22}-S_{2,1}^{T}\right) \\
& \Xi_{13}^{(i 1)}=P_{2} B F_{22}+\sigma_{22} S_{2,1}^{T} \text {, } \\
& \Xi_{14}^{(i 1)}=\sigma_{12} S_{2}^{T} \text {, } \\
& \Xi_{22}^{(i 1)}=\sigma_{12}\left(-2 h_{1} R_{12}+2 h_{12} R_{22}+S_{2}^{T}+S_{2}\right) \\
& +\sigma_{22}\left(-2 R_{22}+S_{2,1}^{T}+S_{2,1}\right) \\
& \Xi_{16}^{(i 1)}=P_{2} E+C^{T} D, \\
& \Xi_{23}^{(i 1)}=\sigma_{22}\left(R_{22}-S_{2,1}^{T}-S_{2,2}^{T}\right) \text {, } \\
& \Xi_{24}^{(i 1)}=\sigma_{12}\left(h_{1} R_{12}-h_{12} R_{22}-S_{2}^{T}\right) \text {, } \\
& \Xi_{25}^{(i 1)}=\sigma_{22} S_{2,2}^{T} \text {, } \\
& \Xi_{33}^{(i 1)}=\sigma_{22}\left(-2 R_{22}+S_{2,2}^{T}+S_{2,2}\right), \\
& \Xi_{35}^{(i 1)}=\sigma_{22}\left(R_{22}-S_{2,2}^{T}\right), \\
& \Xi_{44}^{(i 1)}=\sigma_{12}\left(-h_{1} R_{12}+h_{12} R_{22}\right)+e^{-a_{2} h_{1}}\left(Q_{22}-Q_{12}\right), \\
& \Xi_{55}^{(i 1)}=-\sigma_{22} R_{22}-e^{-a_{2} h_{2}} Q_{22}, \\
& \Xi_{66}^{(i 1)}=-\gamma^{2} I+D^{T} D \\
& \Psi_{2}=\left[\begin{array}{llllll}
A & B F_{12} & B F_{22} & 0 & 0 & E
\end{array}\right], \\
& \sigma_{12}=\frac{e^{-a_{2} h_{1}}}{h_{1}}, \\
& \sigma_{22}=\frac{e^{-a_{2} h_{2}} h_{12}}{h_{2}} \text {, }
\end{aligned}
$$




$$
\begin{aligned}
& \Xi^{(i 2)}=\left[\begin{array}{cccccc}
\Xi_{11}^{(i 2)} & \Xi_{12}^{(i 2)} & \Xi_{13}^{(i 2)} & 0 & 0 & \Xi_{16}^{(i 2)} \\
* & \Xi_{22}^{(i 2)} & \Xi_{23}^{(i 2)} & \Xi_{24}^{(i 2)} & 0 & 0 \\
* & * & \Xi_{33}^{(i 2)} & \Xi_{34}^{(i 2)} & 0 & 0 \\
* & * & * & \Xi_{44}^{(i 2)} & \Xi_{45}^{(i 2)} & 0 \\
* & * & * & * & \Xi_{55}^{(i 2)} & 0 \\
* & * & * & * & * & \Xi_{66}^{(i 2)}
\end{array}\right] \\
& +\Psi_{3}^{T}\left(h_{1}^{2} R_{13}+h_{12}^{2} R_{23}\right) \Psi_{3} \text {, } \\
& \Xi_{11}^{(i 2)}=A^{T} P_{3}+P_{3} A+a_{3} P_{3}+Q_{13}+C^{T} C-\sigma_{13} R_{13}, \\
& a=\min \left\{\lambda_{\min }\left(P_{1}\right), \lambda_{\min }\left(P_{2}\right), \lambda_{\min }\left(P_{3}\right)\right\}, \\
& b=\lambda_{\max }\left(P_{1}\right)+\frac{\left(1-e^{-a_{1} h_{2}}\right)}{a_{1} b_{q}}+\frac{h_{1}\left(1-e^{-a_{1} h_{1}}\right)}{a_{1}^{2} b_{r}} \\
& +\frac{h_{12}\left(e^{-a_{1} h_{1}}-e^{-a_{1} h_{2}}\right)}{a_{1}^{2} b_{r}}, \\
& b_{q}=\max \left\{\lambda_{\max }\left(Q_{11}\right), \lambda_{\max }\left(Q_{21}\right)\right\} \text {, } \\
& b_{r}=\max \left\{\lambda_{\max }\left(R_{11}\right), \lambda_{\max }\left(R_{21}\right)\right\} \text {. }
\end{aligned}
$$$$
\Xi_{12}^{(i 2)}=P_{3} B F_{13}+\sigma_{13} S_{3,1}^{T},
$$$$
\Xi_{13}^{(i 2)}=P_{3} B F_{23}+\sigma_{13}\left(R_{13}-S_{3,1}^{T}\right),
$$$$
\Xi_{16}^{(i 2)}=E+\bar{P}_{3} C^{T} D,
$$$$
\Xi_{22}^{(i 2)}=-\sigma_{13}\left(2 R_{13}-S_{3,2}^{T}-S_{3,2}\right),
$$$$
\Xi_{23}^{(i 2)}=\sigma_{13}\left(R_{13}-S_{3,1}-S_{3,2}\right),
$$$$
\Xi_{24}^{(i 2)}=\sigma_{13}\left(R_{13}-S_{3,2}^{T}\right),
$$$$
\Xi_{33}^{(i 2)}=\sigma_{13}\left(-2 R_{13}+S_{3,1}^{T}+S_{3,1}\right),
$$$$
\Xi_{34}^{(i 2)}=\sigma_{13} S_{3,2}^{T},
$$$$
\Xi_{44}^{(i 2)}=-\sigma_{13} R_{13}-\sigma_{23} R_{23}+\sigma_{13}\left(Q_{23}-Q_{13}\right) \text {, }
$$$$
\Xi_{45}^{(i 2)}=\sigma_{23} R_{23},
$$$$
\Xi_{55}^{(i 2)}=-\sigma_{23}\left(R_{23}+Q_{23}\right),
$$$$
\Xi_{66}^{(i 2)}=-\gamma^{2} I+D^{T} D,
$$$$
\Psi_{3}=\left[\begin{array}{llllll}
A & B F_{13} & B F_{23} & 0 & 0 & E
\end{array}\right],
$$$$
\sigma_{13}=e^{-a_{3} h_{1}},
$$$$
\sigma_{23}=e^{-a_{3} h_{2}} \text {. }
$$

Moreover, the parameters in the exponential stability and $H_{\infty}$ performance are given by

$$
\begin{aligned}
& \alpha=\frac{\left[a_{1}\left(l_{2}-2\right)+a_{2}\left(l_{2}+3\right)-a_{3}\left(l_{2}+1\right)\right]}{l_{2}}, \\
& \beta=\left(\frac{b}{a}\right)^{1 / 2}, \\
& \lambda=\frac{\left[a_{1}\left(l_{2}-2\right)+a_{2}\left(l_{2}+3\right)-a_{3}\left(l_{2}+1\right)\right]}{\left(2 l_{2}\right)}-\frac{\ln \mu}{h_{2}}
\end{aligned}
$$

Proof. See Appendix A.

We now state and establish the delay-dependent criterion for the switched controller design.

Proposition 3. Given $\gamma>0, h_{2}>h_{1}>0, l_{1}>l_{2}>0, v>0$, $0 \leq a_{1} \leq a_{2} \leq a_{3} \leq\left(\left(l_{2}-2\right) a_{1}+\left(l_{2}+3\right) a_{2}\right) /\left(l_{2}+1\right)$, and $\mu \geq 1$ satisfying $\ln (\mu)<\left[\left(a_{1}\left(l_{2}-2\right)+a_{2}\left(l_{2}+3\right)-a_{3}\left(l_{2}+1\right)\right) / l_{2}\right]\left(h_{2} / 2\right)$, system (8) is exponentially stable with a given $H_{\infty}$ performance $\gamma$ for any switching signal with $\ln (\mu)<\left[\left(a_{1}\left(l_{2}-2\right)+a_{2}\left(l_{2}+3\right)-\right.\right.$ $\left.\left.a_{3}\left(l_{2}+1\right)\right) / l_{2}\right]\left(h_{2} / 2\right)$, if there exist matrices $\bar{S}_{1}, \bar{S}_{2}, \bar{S}_{i, 1}, \bar{S}_{i, 2}(i=$ 2, 3), $\bar{F}_{1}, \bar{F}_{12}, \bar{F}_{22}, \bar{F}_{13}$, and $\bar{F}_{23}$ and symmetric matrices $\bar{P}_{j}>0$, $\bar{Q}_{i j}>0$, and $\bar{R}_{i j}>0(i=1,2 ; j=1,2,3)$ such that $\bar{P}_{2} \leq \mu \bar{P}_{1}$, $\bar{P}_{1} \leq \mu \bar{P}_{2}, \bar{P}_{2} \leq \mu \bar{P}_{3}, \bar{P}_{3} \leq \mu \bar{P}_{2}, \bar{Q}_{i 2} \leq \mu \bar{Q}_{i 1}, \bar{Q}_{i 1} \leq \mu \bar{Q}_{i 2}$, $\bar{Q}_{i 2} \leq \mu \bar{Q}_{i 3}, \bar{Q}_{i 3} \leq \mu \bar{Q}_{i 2}, \bar{R}_{i 2} \leq \mu \bar{R}_{i 1}, \bar{R}_{i 1} \leq \mu \bar{R}_{i 2}, \bar{R}_{i 2} \leq \mu \bar{R}_{i 3}$, $\bar{R}_{i 3} \leq \mu \bar{R}_{i 2}(i=1,2)$, and

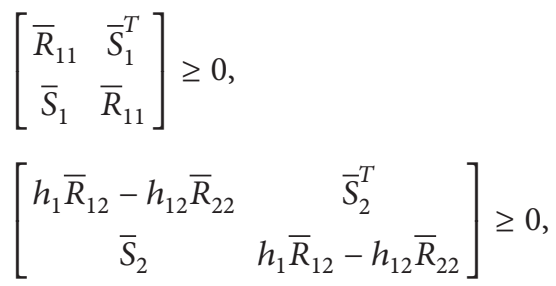

$\left[\begin{array}{ll}\bar{R}_{1 i} & \bar{S}_{i, 1}^{T} \\ \bar{S}_{i, 1} & \bar{R}_{1 i}\end{array}\right] \geq 0, \quad(i=2,3)$,

$\left[\begin{array}{ll}\bar{R}_{1 i} & \bar{S}_{i, 2}^{T} \\ \bar{S}_{i, 2} & \bar{R}_{1 i}\end{array}\right] \geq 0, \quad(i=2,3)$,

$\bar{\Xi}^{(1)}=\left[\begin{array}{cc}\bar{\Pi}_{11}^{(1)} & \bar{\Pi}_{12}^{(1)} \\ * & \bar{\Pi}_{22}^{(1)}\end{array}\right]<0, \quad t \in \mathscr{I}_{1}$,

$\bar{\Xi}^{(i 1)}$

$$
=\left[\begin{array}{cc}
\bar{\Pi}_{11}^{(i 1)} & \bar{\Pi}_{12}^{(i 1)} \\
* & \bar{\Pi}_{22}^{(i 1)}
\end{array}\right]<0,
$$

$t \in \mathscr{I}_{i}, \quad i=m_{v-1}+v+1, \ldots, m_{v}+v, \quad v=1,2, \ldots, l_{2}$, 
$\bar{\Xi}^{(i 2)}$

$$
\begin{aligned}
=\left[\begin{array}{cc}
\bar{\Pi}_{11}^{(i 2)} & \bar{\Pi}_{12}^{(i 2)} \\
* & \bar{\Pi}_{22}^{(i 2)}
\end{array}\right] & <0, \\
& \quad t \in \mathscr{I}_{i}, \quad i=m_{\nu-1}+v, v=2, \ldots, l_{2},
\end{aligned}
$$

where

$$
\bar{\Pi}_{11}^{(1)}=\left[\begin{array}{ccccc}
\bar{\Xi}_{11}^{(1)} & \bar{\Xi}_{12}^{(1)} & \bar{\Xi}_{13}^{(1)} & 0 & E+\bar{P}_{1} C^{T} D \\
* & \bar{\Xi}_{22}^{(1)} & \bar{\Xi}_{23}^{(1)} & 0 & 0 \\
* & * & \bar{\Xi}_{33}^{(1)} & \bar{\Xi}_{34}^{(1)} & 0 \\
* & * & * & \bar{\Xi}_{44}^{(1)} & 0 \\
* & * & * & * & -\gamma^{2} I+D^{T} D
\end{array}\right],
$$$$
\bar{\Pi}_{12}^{(1)}=\left[\begin{array}{ccc}
h_{1} \bar{P}_{1} A^{T} & h_{12} \bar{P}_{1} A^{T} & \bar{P}_{1} C^{T} \\
h_{1} \bar{F}_{1}^{T} B^{T} & h_{12} \bar{F}_{1}^{T} B^{T} & 0 \\
0 & 0 & 0 \\
0 & 0 & 0 \\
h_{1} E^{T} & h_{12} E^{T} & 0
\end{array}\right] \text {, }
$$$$
\bar{\Pi}_{22}^{(1)}=\left[\begin{array}{ccc}
v^{2} \bar{R}_{11}-2 v \bar{P}_{1} & 0 & 0 \\
* & v^{2} \bar{R}_{21}-2 v \bar{P}_{1} & 0 \\
* & * & -I
\end{array}\right] \text {, }
$$$$
\bar{\Xi}_{11}^{(1)}=\bar{P}_{1} A^{T}+A \bar{P}_{1}+a_{1} \bar{P}_{1}+\bar{Q}_{11}-\sigma_{11} \bar{R}_{11} \text {, }
$$$$
\bar{\Xi}_{12}^{(1)}=B \bar{F}_{1}+\sigma_{11}\left(\bar{R}_{11}-\bar{S}_{1}^{T}\right) \text {, }
$$$$
\bar{\Xi}_{13}^{(1)}=\sigma_{11} \overline{\mathrm{S}}_{1}^{T} \text {, }
$$$$
\bar{\Xi}_{22}^{(1)}=-\sigma_{11}\left(2 \bar{R}_{11}-\bar{S}_{1}^{T}-\bar{S}_{1}\right) \text {, }
$$$$
\bar{\Xi}_{23}^{(1)}=\sigma_{11}\left(\bar{R}_{11}-\bar{S}_{1}^{T}\right) \text {, }
$$$$
\bar{\Xi}_{33}^{(1)}=-\sigma_{11} \bar{R}_{11}-\sigma_{21} \bar{R}_{21}+\sigma_{11}\left(\bar{Q}_{21}-\bar{Q}_{11}\right) \text {, }
$$$$
\bar{\Xi}_{34}^{(1)}=\sigma_{21} \bar{R}_{21} \text {, }
$$$$
\bar{\Xi}_{44}^{(1)}=-\sigma_{21}\left(\bar{R}_{21}+\bar{Q}_{21}\right) \text {, }
$$$$
\sigma_{11}=e^{-a_{1} h_{1}},
$$$$
\sigma_{21}=e^{-a_{1} h_{2}} \text {, }
$$

$$
\bar{\Pi}_{11}^{(i 1)}=\left[\begin{array}{cccccc}
\bar{\Xi}_{11}^{(i 1)} & \bar{\Xi}_{12}^{(i 1)} & \bar{\Xi}_{13}^{(i 1)} & \bar{\Xi}_{14}^{(i 1)} & 0 & \bar{\Xi}_{16}^{(i 1)} \\
* & \bar{\Xi}_{22}^{(i 1)} & \bar{\Xi}_{23}^{(i 1)} & \bar{\Xi}_{24}^{(i 1)} & \bar{\Xi}_{25}^{(i 1)} & 0 \\
* & * & \bar{\Xi}_{33}^{(i 1)} & 0 & \bar{\Xi}_{35}^{(i 1)} & 0 \\
* & * & * & \bar{\Xi}_{44}^{(i 1)} & 0 & 0 \\
* & * & * & * & \bar{\Xi}_{55}^{(i 1)} & 0 \\
* & * & * & * & * & \bar{\Xi}_{66}^{(i 1)}
\end{array}\right],
$$

$$
\bar{\Pi}_{12}^{(i 1)}=\left[\begin{array}{ccc}
h_{1} \bar{P}_{2} A^{T} & h_{12} \bar{P}_{2} A^{T} & \bar{P}_{2} C^{T} \\
h_{1} \bar{F}_{12}^{T} B^{T} & h_{12} \bar{F}_{12}^{T} B^{T} & 0 \\
h_{1} \bar{F}_{22}^{T} B^{T} & h_{12} \bar{F}_{22}^{T} B^{T} & 0 \\
0 & 0 & 0 \\
0 & 0 & 0 \\
h_{1} E^{T} & h_{12} E^{T} & 0
\end{array}\right],
$$$$
\bar{\Pi}_{22}^{(i 1)}=\left[\begin{array}{ccc}
v^{2} \bar{R}_{12}-2 v \bar{P}_{2} & 0 & 0 \\
* & v^{2} \bar{R}_{22}-2 v \bar{P}_{2} & 0 \\
* & * & -I
\end{array}\right] \text {, }
$$$$
\bar{\Xi}_{11}^{(i 1)}=\bar{P}_{2} A^{T}+A \bar{P}_{2}+a_{2} \bar{P}_{2}+\bar{Q}_{12}-\sigma_{22} \bar{R}_{22}
$$$$
-\sigma_{12}\left(h_{1} \bar{R}_{12}-h_{12} \bar{R}_{22}\right), \quad h_{12}=h_{2}-h_{1},
$$$$
\bar{\Xi}_{12}^{(i 1)}=B \bar{F}_{12}+\sigma_{12}\left(h_{1} \bar{R}_{12}-h_{12} \bar{R}_{22}-\bar{S}_{2}^{T}\right)
$$$$
+\sigma_{22}\left(\bar{R}_{22}-\bar{S}_{2,1}^{T}\right),
$$

$\bar{\Xi}_{13}^{(i 1)}=B \bar{F}_{22}+\sigma_{22} \bar{S}_{2,1}^{T}$,

$\bar{\Xi}_{14}^{(i 1)}=\sigma_{12} \bar{S}_{2}^{T}$,

$\bar{\Xi}_{16}^{(i 1)}=E+\bar{P}_{2} C^{T} D$,

$\bar{\Xi}_{22}^{(i 1)}=\sigma_{12}\left(-2 h_{1} \bar{R}_{12}+2 h_{12} \bar{R}_{22}+\bar{S}_{2}^{T}+\bar{S}_{2}\right)$

$$
+\sigma_{22}\left(-2 \bar{R}_{22}+\bar{S}_{2,1}^{T}+\bar{S}_{2,1}\right),
$$

$\bar{\Xi}_{23}^{(i 1)}=\sigma_{22}\left(\bar{R}_{22}-\bar{S}_{2,1}^{T}-\bar{S}_{2,2}^{T}\right)$,

$\bar{\Xi}_{24}^{(i 1)}=\sigma_{12}\left(h_{1} \bar{R}_{12}-h_{12} \bar{R}_{22}-\bar{S}_{2}^{T}\right)$,

$\bar{\Xi}_{25}^{(i 1)}=\sigma_{22} \bar{S}_{2,2}^{T}$,

$\bar{\Xi}_{33}^{(i 1)}=\sigma_{22}\left(-2 \bar{R}_{22}+\bar{S}_{2,2}^{T}+\bar{S}_{2,2}\right)$,

$\bar{\Xi}_{35}^{(i 1)}=\sigma_{22}\left(\bar{R}_{22}-\bar{S}_{2,2}^{T}\right)$,

$\bar{\Xi}_{44}^{(i 1)}=\sigma_{12}\left(-h_{1} \bar{R}_{12}+h_{12} \bar{R}_{22}\right)+e^{-a_{2} h_{1}}\left(\bar{Q}_{22}-\bar{Q}_{12}\right)$,

$\bar{\Xi}_{55}^{(i 1)}=-\sigma_{22} \bar{R}_{22}-e^{-a_{2} h_{2}} \bar{Q}_{22}$,

$\bar{\Xi}_{66}^{(i 1)}=-\gamma^{2} I+D^{T} D$,

$\sigma_{12}=\frac{e^{-a_{2} h_{1}}}{h_{1}}$,

$\sigma_{22}=\frac{e^{-a_{2} h_{2}} h_{12}}{h_{2}}$, 


$$
\begin{aligned}
& \bar{\Pi}_{11}^{(i 2)}=\left[\begin{array}{cccccc}
\bar{\Xi}_{11}^{(i 2)} & \bar{\Xi}_{12}^{(i 2)} & \bar{\Xi}_{13}^{(i 2)} & 0 & 0 & \bar{\Xi}_{16}^{(i 2)} \\
* & \bar{\Xi}_{22}^{(i 2)} & \bar{\Xi}_{23}^{(i 2)} & \bar{\Xi}_{24}^{(i 2)} & 0 & 0 \\
* & * & \bar{\Xi}_{33}^{(i 2)} & \bar{\Xi}_{34}^{(i 2)} & 0 & 0 \\
* & * & * & \bar{\Xi}_{44}^{(i 2)} & \bar{\Xi}_{45}^{(i 2)} & 0 \\
* & * & * & * & \bar{\Xi}_{55}^{(i 2)} & 0 \\
* & * & * & * & * & \bar{\Xi}_{66}^{(i 2)}
\end{array}\right], \\
& \bar{\Pi}_{12}^{(i 2)}=\left[\begin{array}{ccc}
h_{1} \bar{P}_{3} A^{T} & h_{12} \bar{P}_{3} A^{T} & \bar{P}_{3} C^{T} \\
h_{1} \bar{F}_{13}^{T} B^{T} & h_{12} \bar{F}_{13}^{T} B^{T} & 0 \\
h_{1} \bar{F}_{23}^{T} B^{T} & h_{12} \bar{F}_{23}^{T} B^{T} & 0 \\
0 & 0 & 0 \\
0 & 0 & 0 \\
h_{1} E^{T} & h_{12} E^{T} & 0
\end{array}\right] \text {, } \\
& \bar{\Pi}_{22}^{(i 2)}=\left[\begin{array}{ccc}
v^{2} \bar{R}_{13}-2 v \bar{P}_{3} & 0 & 0 \\
* & v^{2} \bar{R}_{23}-2 v \bar{P}_{3} & 0 \\
* & * & -I
\end{array}\right] \text {, } \\
& \bar{\Xi}_{11}^{(i 2)}=\bar{P}_{3} A^{T}+A \bar{P}_{3}+a_{3} \bar{P}_{3}+\bar{Q}_{13}-\sigma_{13} \bar{R}_{13} \text {, } \\
& \bar{\Xi}_{12}^{(i 2)}=B \bar{F}_{13}+\sigma_{13} \bar{S}_{3,1}^{T} \text {, } \\
& \bar{\Xi}_{13}^{(i 2)}=B \bar{F}_{23}+\sigma_{13}\left(\bar{R}_{13}-\bar{S}_{3,1}^{T}\right), \\
& \bar{\Xi}_{16}^{(i 2)}=E+\bar{P}_{3} C^{T} D \text {, } \\
& \bar{\Xi}_{22}^{(i 2)}=-\sigma_{13}\left(2 \bar{R}_{13}-\bar{S}_{3,2}^{T}-\bar{S}_{3,2}\right), \\
& \bar{\Xi}_{23}^{(i 2)}=\sigma_{13}\left(\bar{R}_{13}-\bar{S}_{3,1}-\bar{S}_{3,2}\right) \text {, } \\
& \bar{\Xi}_{24}^{(i 2)}=\sigma_{13}\left(\bar{R}_{13}-\bar{S}_{3,2}^{T}\right) \text {, } \\
& \bar{\Xi}_{33}^{(i 2)}=\sigma_{13}\left(-2 \bar{R}_{13}+\bar{S}_{3,1}^{T}+\bar{S}_{3,1}\right), \\
& \bar{\Xi}_{34}^{(i 2)}=\sigma_{13} \bar{S}_{3,2}^{T} \text {, } \\
& \bar{\Xi}_{44}^{(i 2)}=-\sigma_{13} \bar{R}_{13}-\sigma_{23} \bar{R}_{23}+\sigma_{13}\left(\bar{Q}_{23}-\bar{Q}_{13}\right) \text {, } \\
& \bar{\Xi}_{45}^{(i 2)}=\sigma_{23} \bar{R}_{23} \text {, } \\
& \bar{\Xi}_{55}^{(i 2)}=-\sigma_{23}\left(\bar{R}_{23}+\bar{Q}_{23}\right), \\
& \bar{\Xi}_{66}^{(i 2)}=-\gamma^{2} I+D^{T} D, \\
& \sigma_{13}=e^{-a_{3} h_{1}}, \\
& \sigma_{23}=e^{-a_{3} h_{2}} \text {. }
\end{aligned}
$$

Moreover, the control gains of controller (2) are obtained by $F_{1}=\bar{F}_{1} \bar{P}_{1}^{-1}, F_{2}=\bar{F}_{2} \bar{P}_{2}^{-1}$, and $F_{3}=\bar{F}_{3} \bar{P}_{3}^{-1}$, where $\bar{F}_{2}=\bar{F}_{12}+$ $\bar{F}_{22}$ and $\bar{F}_{3}=\bar{F}_{13}+\bar{F}_{23}$. The parameters in the exponential stability and $H_{\infty}$ performance are given by

$$
\begin{aligned}
& \alpha=\frac{\left[a_{1}\left(l_{2}-2\right)+a_{2}\left(l_{2}+3\right)-a_{3}\left(l_{2}+1\right)\right]}{l_{2}}, \\
& \beta=\left(\frac{\bar{b}}{\bar{a}}\right)^{1 / 2}, \\
& \lambda=\frac{\left[a_{1}\left(l_{2}-2\right)+a_{2}\left(l_{2}+3\right)-a_{3}\left(l_{2}+1\right)\right]}{\left(2 l_{2}\right)}-\frac{\ln \mu}{h_{2}}
\end{aligned}
$$

with

$$
\begin{aligned}
\bar{a}= & \min \left\{\lambda_{\min }\left(\bar{P}_{1}^{-1}\right), \lambda_{\min }\left(\bar{P}_{2}^{-1}\right), \lambda_{\min }\left(\bar{P}_{3}^{-1}\right)\right\}, \\
\bar{b}= & \lambda_{\max }\left(\bar{P}_{1}^{-1}\right)+\frac{\left(1-e^{-a_{1} h_{2}}\right)}{a_{1} \bar{b}_{q}}+\frac{h_{1}\left(1-e^{-a_{1} h_{1}}\right)}{a_{1}^{2} \bar{b}_{r}} \\
& +\frac{h_{12}\left(e^{-a_{1} h_{1}}-e^{-a_{1} h_{2}}\right)}{a_{1}^{2} \bar{b}_{r}}, \\
\bar{b}_{q}= & \max \left\{\lambda_{\max }\left(\bar{P}_{1}^{-1} \bar{Q}_{11} \bar{P}_{1}^{-1}\right), \lambda_{\max }\left(\bar{P}_{1}^{-1} \bar{Q}_{21} \bar{P}_{1}^{-1}\right)\right\}, \\
\bar{b}_{r}= & \max \left\{\lambda_{\max }\left(\bar{P}_{1}^{-1} \bar{R}_{11} \bar{P}_{1}^{-1}\right), \lambda_{\max }\left(\bar{P}_{1}^{-1} \bar{R}_{21} \bar{P}_{1}^{-1}\right)\right\} .
\end{aligned}
$$

Proof. See Appendix B.

Remark 4. Suppose that the system matrices $\Omega:=\left[\begin{array}{ll}A & B\end{array}\right]$ are not exactly known and they reside in the uncertain polytope $[16,17,26]$. Consider

$$
\Omega \in\left\{\sum_{i=1}^{M} \mu_{i} \Omega_{i}, 0 \leq \mu_{i} \leq 1, \sum_{i=1}^{M} \mu_{i}=1\right\}
$$

where the $M$ vertices of the polytope are described by $\Omega_{i}:=\left[\begin{array}{ll}A_{i} & B_{i}\end{array}\right]$. For the $H_{\infty}$ performance analysis and the periodic switched controller design of the dual-rate system with polytopic uncertainties, one can solve the LMIs in Propositions 2 and 3 for all the $M$ vertices $\Omega_{i}$ by applying the same decision matrices, respectively.

For comparison purpose, a criterion for the existence of a sampled-data controller for system (1) via single-rate sampling is developed and given by the following corollary.

Corollary 5. Given $h>0, a>0$, and $v>0$, system (1) with a single-rate sampled-data controller is exponentially stable with a given $H_{\infty}$ performance $\gamma$, where the exponential decay rate 
$\alpha=a$, if there exist a matrix $\bar{S}$ and symmetric matrices $\bar{P}>$ $0, \bar{Q}>0$, and $\bar{R}>0$ such that

$$
\bar{\Xi}=\left[\begin{array}{cccccc}
\bar{\Xi}_{11} & \bar{\Xi}_{12} & \sigma \bar{S}^{T} & \bar{\Xi}_{14} & h \bar{P} A^{T} & \bar{P} C^{T} \\
* & \bar{\Xi}_{22} & \bar{\Xi}_{23} & 0 & h \bar{F}^{T} B^{T} & 0 \\
* & * & \bar{\Xi}_{33} & 0 & 0 & 0 \\
* & * & * & \bar{\Xi}_{44} & h E^{T} & 0 \\
* & * & * & * & \bar{\Xi}_{55} & 0 \\
* & * & * & * & * & -I
\end{array}\right]<0,
$$

where

$$
\begin{aligned}
\bar{\Xi}_{11} & =\bar{P} A^{T}+A \bar{P}+a \bar{P}+\bar{Q}-\sigma \bar{R}, \\
\bar{\Xi}_{12} & =B \bar{F}+\sigma\left(\bar{R}-\bar{S}^{T}\right), \\
\bar{\Xi}_{14} & =E+\bar{P} C^{T} D, \\
\bar{\Xi}_{22} & =\sigma\left(-2 \bar{R}+\bar{S}^{T}+\bar{S}\right), \\
\bar{\Xi}_{23} & =\sigma\left(\bar{R}-\bar{S}^{T}\right), \\
\bar{\Xi}_{33} & =-\sigma(\bar{R}+\bar{Q}), \\
\bar{\Xi}_{44} & =-\gamma^{2} I+D^{T} D, \\
\bar{\Xi}_{55} & =v^{2} \bar{R}-2 v \bar{P}, \\
\sigma & =e^{-a h} .
\end{aligned}
$$

Moreover, the control gain is obtained as $F=\bar{F} \bar{P}^{-1}$.

Remark 6. For the case $l_{1}=N l_{2}(N>1$ and $N \in \mathbb{N})$, periodic switched controller (2) reduces to a switched controller with switching modes $\sigma(t)=1$ and $\sigma(t)=2$. Correspondingly, Proposition 3 with $\bar{P}_{3}=0, \bar{Q}_{i 3}=0, \bar{S}_{3, i}=0$, and $\bar{R}_{i 3}=0$ $(i=1,2)$ reduces to a sampled-data $H_{\infty}$ control design result for system (1) via two sampling rates $1 / h_{1}$ and $1 / N h_{1}$. Particularly, when $N=2$, the design result can be reduced to the one in [28].

Remark 7. In this paper, we obtain some results on periodic switched control of dual-rate sampled-data systems. It should be pointed out that these results can be extended to multirate sampled-data systems although the process is very tedious. The corresponding results are omitted.

Remark 8. As shown in $[20,29]$, analysis and synthesis of research on networked control systems with multiple samplings are promising and significant issues. Compared with [20], this paper is distinguished based on the following evidences: (i) The dual-rate sampling schemes in this paper and [20] are different, resulting in two essentially different switched system models. In [20], by introducing a Round-Robin scheduling protocol, the sampled-data via two different sampling rates is available one after another. In this paper, however, the sampled-data is available in three cases (both $x_{1}\left(k h_{1}\right)$ and $x_{2}\left(k h_{2}\right)$, only $x_{1}\left(k h_{1}\right)$, and/or only $\left.x_{2}\left(k h_{2}\right)\right)$. In other words, under the sampling relationship $l_{1} h_{1}=l_{2} h_{2}$ in this paper, the case that $x_{1}\left(k h_{1}\right)$ and $x_{2}\left(k h_{2}\right)$ are available simultaneously must occur, but this surely does not occur in [20]. (ii) The problems addressed in this paper and [20] are different. Stability and $L_{2}$-gain analysis carried out for dual-rate networked control systems is considered in [20], while this paper focuses on controller design for a dual-rate sampled-data system without considering the effect of network-induced delays. It should be mentioned that the constant constraint on networkinduced delays in [20] does not significantly change the nature of the proposed results. (iii) The methodologies of performance analysis in this paper and [20] are different. In [20], a common discontinuous Lyapunov-Krasovskii functional is constructed to derive some delay-dependent stability and $L_{2}$-gain criteria. However, in this paper, a switched continuous Lyapunov-Krasovskii functional is proposed to establish delay-dependent criteria for the $H_{\infty}$ performance and switched controller design by fully utilizing the relation of multiple input delays and the information about two sampling periods.

\section{The Dual-Rate Sampled-Data Control of a Vehicle Dynamic System}

In this section, we consider the dual-rate sampled-data control of a vehicle dynamic system described by the following state-space representation [30]:

$$
\begin{aligned}
& \dot{x}(t)=A x(t)+B u(t)+E \omega(t), \\
& z(t)=C x(t)+D \omega(t)
\end{aligned}
$$

where

$$
\begin{aligned}
& A=\left[\begin{array}{cccc}
0 & 1 & 0 & 22.3 \\
0 & -4.2796 & -19.4355 & 0 \\
0 & 1.4391 & -4.2743 & 0 \\
0 & 0 & 1 & 0
\end{array}\right] \text {, } \\
& B=\left[\begin{array}{c}
0 \\
25.0655 \\
17.7548 \\
0
\end{array}\right] \text {, } \\
& C=\left[\begin{array}{llll}
0 & 1 & 0 & 0
\end{array}\right], \\
& D=0.1 \text {, } \\
& E=\left[\begin{array}{llll}
0 & 0.1 & 0.1 & 0
\end{array}\right]^{T},
\end{aligned}
$$

$x(t)=\left[\begin{array}{llll}y & \phi & r & \psi\end{array}\right]^{T}, y$ is the inertial lateral displacement of the vehicle mass center, $\phi$ is lateral velocity in the vehicle body axis system, $r$ is the angular velocity, and $\psi$ is the vehicle heading angle. 
TABLE 1: Solvability comparison between Proposition 3 with $3 h_{1}=$ $2 h_{2}$ and Corollary 5 with $h=h_{2}$.

\begin{tabular}{lcccc}
\hline$h_{2}$ & $0.24 \mathrm{~s}$ & $0.27 \mathrm{~s}$ & $0.30 \mathrm{~s}$ & $0.36 \mathrm{~s}$ \\
\hline $\begin{array}{l}\text { Proposition } 3 \\
\text { (dual-rate) }\end{array}$ & Feasible & Feasible & Feasible & Feasible \\
\hline $\begin{array}{l}\text { Corollary 5 } \\
\text { (single-rate) }\end{array}$ & Feasible & Feasible & Infeasible & Infeasible \\
\hline
\end{tabular}

The state variables $y$ and $\phi$ are measured by using laser sensors at a sampling rate $1 / h_{1}$, and the state variables $r$ and $\psi$ are measured by using gyrometers at a sampling rate $1 / h_{2}$, where $h_{i}(i=1,2)$ are two sampling periods.

We now show the effectiveness of the proposed dual-rate sampling design result. Set $a_{1}=0.75, a_{2}=0.85, a_{3}=$ $0.9, v=0.25, l_{1}=3, l_{2}=2, \mu=1.01, h_{1}=0.16 \mathrm{~s}$, and $h_{2}=0.24 \mathrm{~s}$. Using Proposition 3, we can obtain the minimum $H_{\infty}$ performance $\gamma_{\min }^{d}=0.1539$, an exponential decay rate $\alpha=0.775$, and corresponding control gain matrices $F_{1}=\left[\begin{array}{llll}-0.0141 & -0.0487 & -0.0532 & -0.7625\end{array}\right]$, $F_{2}=\left[\begin{array}{llll}-0.0142 & -0.0492 & -0.0539 & -0.7672\end{array}\right]$, and $F_{3}=$ $\left[\begin{array}{lll}-0.0142-0.0490-0.0546-0.7686\end{array}\right]$, which means that switched controller (2) with the above $F_{i}(i=1,2,3)$ can stabilize system (39) and achieve an $H_{\infty}$ performance $\gamma_{\min }^{d}=0.1539$ and $\alpha=0.775$ for system (39) with an external disturbance. Using Corollary 5 , the minimum $H_{\infty}$ performance $\gamma_{\min }^{s}=0.2317$, the exponential decay rate $a=\alpha=0.775$, and the control gain is $F=[-0.0092-$ $0.0252-0.0386-0.6028]$. Clearly, Proposition 3 can be used to search for a better $H_{\infty}$ control performance than Corollary 5. For comparison purpose, we choose different delay bounds of $h_{2}$ for both dual-rate sampling case and single-rate sampling case. The solvability of Proposition 3 and Corollary 5 is shown in Table 1 , from which one can see that the dual-rate sampling design result can provide a larger sampling period than the single-rate design result.

Then we compare the proposed dual-rate sampling design result and the single-rate design result in simulation. Choose the initial state $x_{0}=\left[\begin{array}{cccc}-2 & 0 & 1 & 0\end{array}\right]^{T}$ and the external disturbance $\omega(t)=2 e^{-0.3 t} \sin (2.7 t+0.05)$. Using the switched controller with $F_{i}(i=1,2,3)$, we can depict the state responses of system (39) by Figure 3 and the corresponding control input by Figure 4, respectively. Under the zero initial condition, $\mathscr{L}_{2}$-norm $\left\|z_{d}(t)\right\|_{2}$ using the switched controller and $\left\|z_{s}(t)\right\|_{2}$ using the single-rate sampled-data controller are shown in Figure 5. From Figures 3-5, one can conclude that the dual-rate sampled-data can stabilize the system (39) and achieve a better $H_{\infty}$ performance than the single-rate sampled-data controller.

\section{Conclusion}

This paper has dealt with the $H_{\infty}$ sampled-data control for a multirate system with two sampling rates by using an input delay approach. A periodic switched controller has been proposed to implement the sampled-data control such that the sampled-data of state variables with different sampling rates

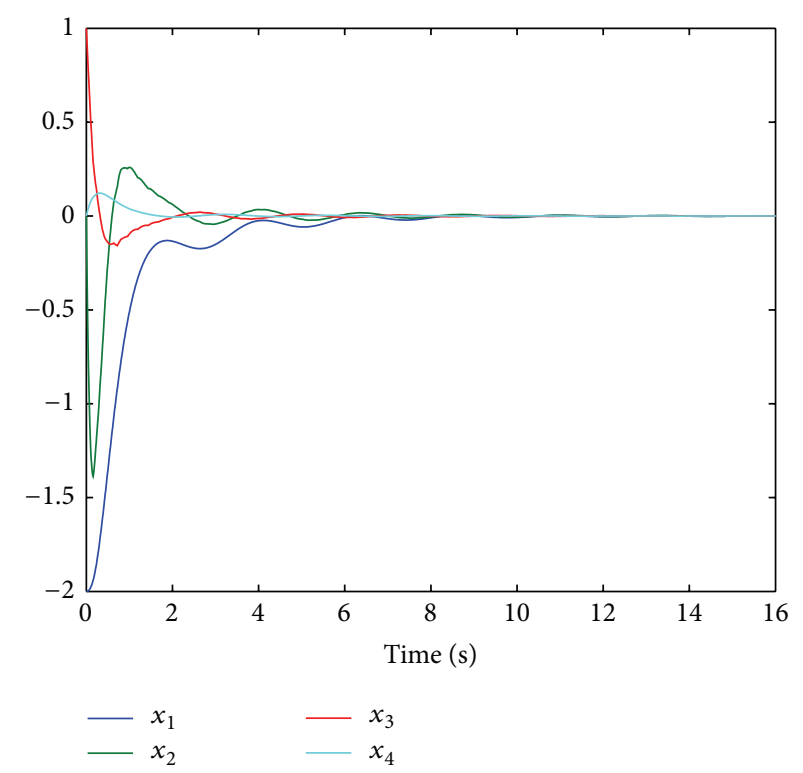

FIgURE 3: The state responses of system (39) using the switched controller.

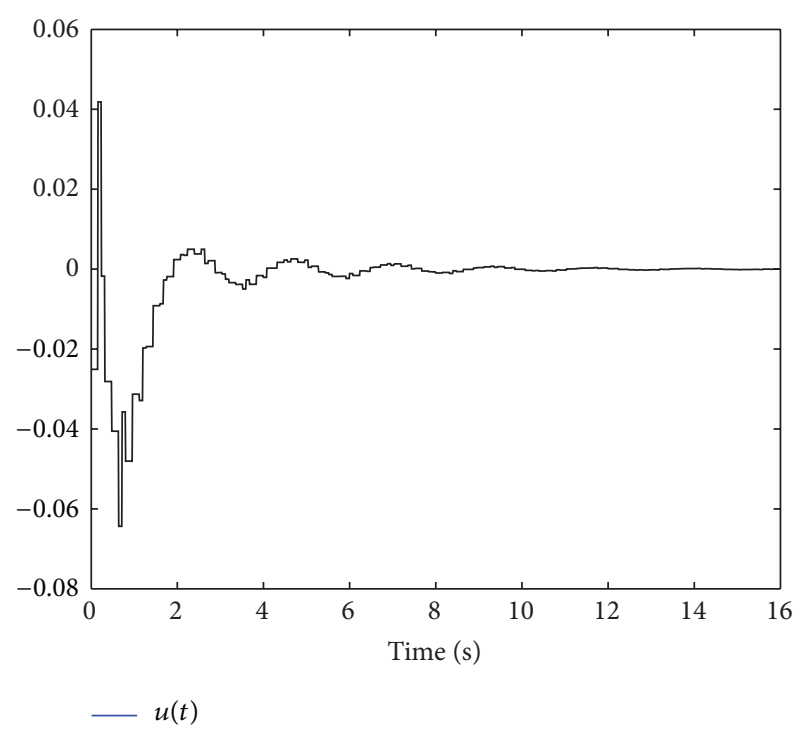

Figure 4: The control input of system (39) using the switched controller.

can be used real-timely. The resulting closed-loop system has been modeled as a switched system, where the subsystems have different input delays. Some delay-dependent criteria for the $H_{\infty}$ performance of the switched system and the existence of the switched controller have been established by using the Lyapunov-Krasovskii functional method. By comparing with single-rate design methods, the effectiveness of the proposed design method has been illustrated by an example. 


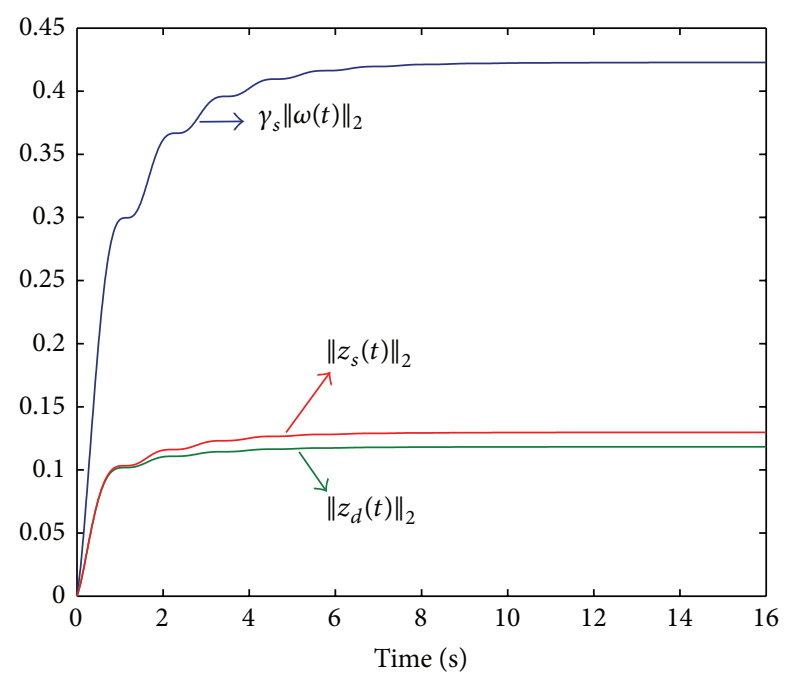

FIGURE 5: The comparison of $\left\|e^{-\alpha t / 2} z(t)\right\|_{2} \leq \gamma\|\omega(t)\|_{2}$ under the zero initial condition by two different methods.

\section{Appendices}

\section{A. Proof of Proposition 2}

Proof. Construct the following Lyapunov-Krasovskii functional:

$$
\begin{aligned}
V_{\sigma(t)}( & t) \\
= & x^{T}(t) P_{\sigma(t)} x(t) \\
& +\int_{t-h_{1}}^{t} x^{T}(s) e^{a_{\sigma(t)}(s-t)} Q_{1 \sigma(t)} x(s) d s \\
& +\int_{t-h_{2}}^{t-h_{1}} x^{T}(s) e^{a_{\sigma(t)}(s-t)} Q_{2 \sigma(t)} x(s) d s \\
& +h_{1} \int_{-h_{1}}^{0} \int_{t+\theta}^{t} \dot{x}^{T}(s) e^{a_{\sigma(t)}(s-t)} R_{1 \sigma(t)} \dot{x}(s) d s d \theta \\
& +h_{12} \int_{-h_{2}}^{-h_{1}} \int_{t+\theta}^{t} \dot{x}^{T}(s) e^{a_{\sigma(t)}(s-t)} R_{2 \sigma(t)} \dot{x}(s) d s d \theta,
\end{aligned}
$$

where $P_{\sigma(t)}>0, Q_{i \sigma(t)}>0$, and $R_{i \sigma(t)}>0(i=1,2, \sigma(t)=$ $1,2,3)$. have

When $\sigma(t)=1$, taking the time derivative of $V_{1}(t)$, we

$$
\begin{aligned}
\dot{V}_{1}(t)= & 2 x^{T}(t) P_{1} \dot{x}(t)+a_{1} x^{T}(t) P_{1} x(t) \\
& +x^{T}(t) Q_{11} x(t)
\end{aligned}
$$

$$
\begin{aligned}
& +e^{-a_{1} h_{1}} x^{T}\left(t-h_{1}\right)\left(Q_{21}-Q_{11}\right) x\left(t-h_{1}\right) \\
& -e^{-a_{1} h_{2}} x^{T}\left(t-h_{2}\right) Q_{21} x\left(t-h_{2}\right) \\
& +\dot{x}^{T}(t)\left(h_{1}^{2} R_{11}+h_{12}^{2} R_{21}\right) \dot{x}(t)-a_{1} V_{1}(t) \\
& -h_{1} \int_{t-h_{1}}^{t} \dot{x}^{T}(s) e^{a_{1}(s-t)} R_{11} \dot{x}(s) d s \\
& -h_{12} \int_{t-h_{2}}^{t-h_{1}} \dot{x}^{T}(s) e^{a_{1}(s-t)} R_{21} \dot{x}(s) d s \\
& \leq-a_{1} V_{1}(t)+2 x^{T}(t) P_{1} \dot{x}(t) \\
& +x^{T}(t)\left(a_{1} P_{1}+Q_{11}\right) x(t) \\
& +\dot{x}^{T}(t)\left(h_{1}^{2} R_{11}+h_{12}^{2} R_{21}\right) \dot{x}(t) \\
& +e^{-a_{1} h_{1}} x^{T}\left(t-h_{1}\right)\left(Q_{21}-Q_{11}\right) x\left(t-h_{1}\right) \\
& -e^{-a_{1} h_{2}} x^{T}\left(t-h_{2}\right) Q_{21} x\left(t-h_{2}\right) \\
& -h_{1} e^{-a_{1} h_{1}} \int_{t-h_{1}}^{t} \dot{x}^{T}(s) R_{11} \dot{x}(s) d s \\
& -h_{12} e^{-a_{1} h_{2}} \int_{t-h_{2}}^{t-h_{1}} \dot{x}^{T}(s) R_{21} \dot{x}(s) d s .
\end{aligned}
$$

For system (8) with $\omega(t) \equiv 0$, by using Lemma 1 and Jensen's inequality to (A.2), it can be derived that $\dot{V}_{1}(t) \leq$ $-a_{1} V_{1}(t)+\widetilde{\xi}_{1}^{T}(t) \widetilde{\Xi}^{(1)} \widetilde{\xi}_{1}(t)$, where

$\tilde{\xi}_{1}^{T}(t)$

$$
\begin{aligned}
& =\left[\begin{array}{llll}
x^{T}(t) & x^{T}\left(t-\tau_{1}(t)\right) & x^{T}\left(t-h_{1}\right) & x^{T}\left(t-h_{2}\right)
\end{array}\right], \\
& \widetilde{\Xi}^{(1)}=\left[\begin{array}{cccc}
\Xi_{11}^{(1)} & \Xi_{12}^{(1)} & \Xi_{13}^{(1)} & 0 \\
* & \Xi_{22}^{(1)} & \Xi_{23}^{(1)} & 0 \\
* & * & \Xi_{33}^{(1)} & \Xi_{34}^{(1)} \\
* & * & * & \Xi_{44}^{(1)}
\end{array}\right]+\left[\begin{array}{llll}
A & B F_{1} & 0 & 0
\end{array}\right]^{T} \\
& \cdot\left(h_{1}^{2} R_{11}+h_{12}^{2} R_{21}\right)\left[\begin{array}{llll}
A & B F_{1} & 0 & 0
\end{array}\right] .
\end{aligned}
$$

If the inequality $\widetilde{\Xi}^{(1)}<0$ holds, then we obtain $\dot{V}_{1}(t) \leq$ $-a_{1} V_{1}(t)$. On the other hand, $\widetilde{\Xi}^{(1)}<0$ is implied by $(20)$.

For the cases $\sigma(t)=2$ and $\sigma(t)=3$, similar to the above process, one can obtain from (21) and (22) that $\dot{V}_{2}(t) \leq$ $-a_{2} V_{2}(t)$ and $\dot{V}_{3}(t) \leq-a_{3} V_{3}(t)$, respectively. 
On different subintervals $\mathscr{I}_{i}\left(i=1,2, \ldots, m_{l_{2}}+l_{2}\right)$, it can be obtained that

$$
\begin{array}{r}
V_{1}(t) \leq e^{-a_{1}\left(t-k l_{1} h_{1}\right)} V_{1}\left(k l_{1} h_{1}\right), \quad t \in \mathscr{I}_{1}, \\
V_{2}(t) \leq e^{-a_{2}\left(t-\left(k l_{1}+m_{\nu-1}+1\right) h_{1}\right)} V_{2}\left(\left(k l_{1}+m_{\nu-1}+1\right) h_{1}\right), \\
t \in \bigcup_{m_{\nu-1}+\nu+1}^{m_{\nu}+v} \mathscr{I}_{i}, v=1,2, \ldots, l_{2}, \\
V_{3}(t) \leq e^{-a_{3}\left(t-k l_{1} h_{1}-(\nu-1) h_{2}\right)} V_{3}\left(k l_{1} h_{1}+(\nu-1) h_{2}\right), \\
t \in \mathscr{I}_{m_{\nu-1}+v}, v=2,3, \ldots, l_{2} .
\end{array}
$$

For $P_{2} \leq \mu P_{1}, P_{1} \leq \mu P_{2}, P_{2} \leq \mu P_{3}, P_{3} \leq \mu P_{2}, Q_{i 2} \leq \mu Q_{i 1}$, $Q_{i 1} \leq \mu Q_{i 2}, Q_{i 2} \leq \mu Q_{i 3}, Q_{i 3} \leq \mu Q_{i 2}, R_{i 2} \leq \mu R_{i 1}, R_{i 1} \leq \mu R_{i 2}$, $R_{i 2} \leq \mu R_{i 3}$, and $R_{i 3} \leq \mu R_{i 2}(i=1,2)$, one has

$$
\begin{aligned}
& V_{1}(t) \leq \mu e^{\left(a_{2}-a_{1}\right) h_{2}} V_{2}(t), \\
& V_{2}(t) \leq \mu e^{\left(a_{3}-a_{2}\right) h_{2}} V_{3}(t), \\
& V_{2}(t) \leq \mu V_{1}(t), \\
& V_{3}(t) \leq \mu V_{2}(t) .
\end{aligned}
$$

Defining $v_{1}=\mu e^{\left(a_{2}-a_{1}\right) h_{2}}$ and $v_{2}=\mu e^{\left(a_{3}-a_{2}\right) h_{2}}$, we obtain

$$
\begin{aligned}
& V_{\sigma\left(t^{+}\right)} \leq v_{1} V_{\sigma\left(t^{-}\right)}, \quad t=k l_{1} h_{1}, \\
& V_{\sigma\left(t^{+}\right)} \leq \mu V_{\sigma\left(t^{-}\right)}, \quad t=k l_{1} h_{1}+h_{1}, k l_{1} h_{1}+i h_{2}, \\
& V_{\sigma\left(t^{+}\right)} \leq v_{2} V_{\sigma\left(t^{-}\right)}, \quad t=k l_{1} h_{1}+\left(m_{i}+1\right) h_{1},
\end{aligned}
$$

where $i=1,2, \ldots, l_{2}-1, k=0,1,2, \ldots$.

For $t \in\left[k l_{1} h_{1}, k l_{1} h_{1}+h_{1}\right)$, using (A.4) and (A.6), we have

$$
\begin{aligned}
& V_{1}(t) \leq v_{1} e^{-a_{1}\left(t-k l_{1} h_{1}\right)} V_{2}\left(k l_{1} h_{1}-0\right) \\
& \quad \leq v_{1} v_{2} e^{-a_{1}\left(t-k l_{1} h_{1}\right)} e^{-a_{2}\left(l_{1} h_{1}-\left(m_{l_{2}-1}+1\right) h_{1}\right)} V_{3}((k-1) \\
& \left.\cdot l_{1} h_{1}+\left(m_{l_{2}-1}+1\right) h_{1}-0\right) \\
& \quad \leq v_{1} v_{2} \mu e^{-a_{1}\left(t-k l_{1} h_{1}\right)} V_{2}\left((k-1) l_{1} h_{1}+\left(l_{2}-1\right) h_{1}\right. \\
& -0) e^{-a_{2}\left(l_{1} h_{1}-\left(m_{l_{2}-1}+1\right) h_{1}\right)-a_{3}\left(\left(m_{l_{2}-1}+1\right) h_{1}-\left(l_{2}-1\right) h_{2}\right)} \leq \cdots \\
& \quad \leq v_{1} v_{2}^{l_{2}-1} \mu^{l_{2}} e^{-a_{1}\left(t-k l_{1} h_{1}\right)} e^{\bar{a}} V_{1}\left((k-1) l_{1} h_{1}\right) \leq \cdots \\
& \quad \leq\left(v_{1} v_{2}^{l_{2}-1} \mu^{l_{2}}\right)^{k} e^{-a_{1}\left(t-k l_{1} h_{1}\right)} e^{k \bar{a}} V_{1}(0),
\end{aligned}
$$

where

$$
\begin{aligned}
\bar{a}= & -a_{2}\left(\left(l_{1}-m_{l_{2}-1}-1\right) h_{1}\right) \\
& -a_{3}\left(\left(m_{l_{2}-1}+1\right) h_{1}-\left(l_{2}-1\right) h_{2}\right) \\
& -a_{2}\left(\left(l_{2}-1\right) h_{2}-\left(m_{l_{2}-2}+1\right) h_{1}\right)
\end{aligned}
$$

$$
\begin{aligned}
& -a_{3}\left(\left(m_{l_{2}-2}+1\right) h_{1}-\left(l_{2}-2\right) h_{2}\right)-\cdots \\
& -a_{2}\left(h_{2}-h_{1}\right)-a_{1} h_{1} .
\end{aligned}
$$

Using $k l_{1} h_{1} \leq t$ to (A.7), one can see that

$V_{1}(t)$

$$
\begin{aligned}
& \leq\left[\mu^{2 l_{2}} e^{\left(a_{2}-a_{1}\right) h_{2}+\left(l_{2}-1\right)\left(a_{3}-a_{2}\right) h_{2}}\right]^{k} e^{-a_{1}\left(t-k l_{1} h_{1}\right)} e^{k \bar{a}} V_{1}(0) \\
& \leq\left[\mu^{2 l_{2}} e^{\left(a_{2}-a_{1}\right) h_{2}+\left(l_{2}-1\right)\left(a_{3}-a_{2}\right) h_{2}+\bar{a}}\right]^{k} e^{-a_{1}\left(t-k l_{1} h_{1}\right)} V_{1}(0) \\
& \leq\left[\mu^{2 / h_{2}} e^{\left[a_{3}\left(l_{2}+1\right)-a_{2}\left(l_{2}+3\right)-a_{1}\left(l_{2}-2\right)\right] / l_{2}}\right]^{t} V_{1}(0) .
\end{aligned}
$$

For $t \in\left[k l_{1} h_{1}+h_{1}, k l_{1} h_{1}+h_{2}\right)$, it is clear that

$$
\begin{aligned}
& V_{2}(t) \leq\left(v_{1} v_{2}^{l_{2}-1} \mu^{l_{2}}\right)^{k} e^{-a_{2}\left(t-k l_{1} h_{1}-h_{1}\right)} e^{k \bar{a}} \mu e^{-a_{1} h_{1}} V_{1}(0) \\
& \leq\left[\mu^{2 l_{2}} e^{\left(a_{2}-a_{1}\right) h_{2}+\left(l_{2}-1\right)\left(a_{3}-a_{2}\right) h_{2}}\right]^{k} \\
& \cdot e^{-a_{2}\left(t-k l_{1} h_{1}-h_{1}\right)} e^{k \bar{a}} \mu e^{-a_{1} h_{1}} V_{1}(0) \\
& \quad \leq\left[\mu^{2 / h_{2}} e^{\left[a_{3}\left(l_{2}+1\right)-a_{2}\left(l_{2}+3\right)-a_{1}\left(l_{2}-2\right)\right] / l_{2}}\right]^{k l_{1} h_{1}+h_{1}} \\
& \cdot e^{-a_{2}\left(t-k l_{1} h_{1}-h_{1}\right)} V_{1}(0) \\
& \quad \leq\left[\mu^{2 / h_{2}} e^{\left[a_{3}\left(l_{2}+1\right)-a_{2}\left(l_{2}+3\right)-a_{1}\left(l_{2}-2\right)\right] / l_{2}}\right]^{t} V_{1}(0)
\end{aligned}
$$

For $t \in\left[k l_{1} h_{1}+h_{2},\left(k l_{1}+m_{1}+1\right) h_{1}\right)$, it can be seen that

$$
\begin{aligned}
& V_{3}(t) \leq\left(v_{1} v_{2}^{l_{2}-1} \mu^{l_{2}}\right)^{k} \\
& \cdot e^{-a_{3}\left(t-k l_{1} h_{1}-h_{2}\right)} e^{k \bar{a}} e^{-a_{2}\left(h_{2}-h_{1}\right)-a_{1} h_{1}} \mu^{2} V_{1}(0) \\
& \leq\left(\mu^{2 l_{2}} e^{\left(a_{2}-a_{1}\right) h_{2}+\left(l_{2}-1\right)\left(a_{3}-a_{2}\right) h_{2}}\right)^{k} \\
& \cdot e^{-a_{3}\left(t-k l_{1} h_{1}-h_{2}\right)} e^{k \bar{a}} e^{-a_{2}\left(h_{2}-h_{1}\right)-a_{1} h_{1}} \mu^{2} V_{1}(0) \\
& \leq\left(\mu^{2 / h_{2}} e^{\left[a_{3}\left(l_{2}+1\right)-a_{2}\left(l_{2}+3\right)-a_{1}\left(l_{2}-2\right)\right] / l_{2}}\right)^{k l_{1} h_{1}+h_{2}} \\
& \cdot e^{-a_{3}\left(t-k l_{1} h_{1}-h_{2}\right)} V_{1}(0) \\
& \leq\left(\mu^{2 / h_{2}} e^{\left[a_{3}\left(l_{2}+1\right)-a_{2}\left(l_{2}+3\right)-a_{1}\left(l_{2}-2\right)\right] / l_{2}}\right)^{t} V_{1}(0) .
\end{aligned}
$$


For $t \in\left[k l_{1} h_{1}+\left(l_{2}-1\right) h_{2},\left(k l_{1}+m_{l_{2}-1}+1\right) h_{1}\right)$, one has

$$
\begin{aligned}
V_{3}(t) & \leq\left(v_{1} v_{2}^{l_{2}-1} \mu^{l_{2}}\right)^{k} e^{-a_{3}\left(t-k l_{1} h_{1}-\left(l_{2}-1\right) h_{2}\right)} e^{k \bar{a}} v_{2}^{l_{2}-2} \mu^{l^{2}} e^{-a_{2}\left(\left(l_{2}-1\right) h_{2}-\left(m_{l_{2}-2}+1\right) h_{1}\right)-\cdots-a_{2}\left(h_{2}-h_{1}\right)-a_{1} h_{1}} V_{1}(0) \\
& \leq\left(\mu^{2 / h_{2}} e^{\left(a_{3}\left(l_{2}+1\right)-a_{2}\left(l_{2}+3\right)-a_{1}\left(l_{2}-2\right)\right] / l_{2}}\right)^{k l_{1} h_{1}+\left(l_{2}-1\right) h_{2}} e^{-a_{3}\left(t-k l_{1} h_{1}-\left(l_{2}-1\right) h_{2}\right)} V_{1}(0) \\
& \leq\left(\mu^{2 / h_{2}} e^{\left(a_{3}\left(l_{2}+1\right)-a_{2}\left(l_{2}+3\right)-a_{1}\left(l_{2}-2\right)\right) / l_{2}}\right)^{t} V_{1}(0) .
\end{aligned}
$$

For $t \in\left[\left(k l_{1}+m_{l_{2}-1}+1\right) h_{1}, k l_{1} h_{1}+l_{2} h_{2}\right)$, one has

$$
\begin{aligned}
& V_{2}(t) \leq\left(v_{1} v_{2}^{l_{2}-1} \mu^{l^{2}}\right)^{k} e^{-a_{2}\left(t-k l_{1} h_{1}-\left(m_{l_{2}-1}+1\right) h_{1}\right)} e^{k \bar{a}} v_{2}^{l_{2}-1} \mu^{l_{2}} e^{-a_{3}\left(\left(m_{l_{2}-1}+1\right) h_{1}-\left(l_{2}-1\right) h_{2}\right)-\cdots-a_{2}\left(h_{2}-h_{1}\right)-a_{1} h_{1}} V_{1}(0) \\
& \quad \leq\left(\mu^{2 / h_{2}} e^{\left(a_{3}\left(l_{2}+1\right)-a_{2}\left(l_{2}+3\right)-a_{1}\left(l_{2}-2\right)\right) / l_{2}}\right)^{k l_{1} h_{1}+\left(m_{l_{2}-1}+1\right) h_{1}} e^{-a_{2}\left(t-k l_{1} h_{1}-\left(m_{l_{2}-1}+1\right) h_{1}\right)} V_{1}(0) \\
& \leq\left(\mu^{2 / h_{2}} e^{\left(a_{3}\left(l_{2}+1\right)-a_{2}\left(l_{2}+3\right)-a_{1}\left(l_{2}-2\right)\right) / l_{2}}\right)^{t} V_{1}(0) .
\end{aligned}
$$

Denote $\varepsilon=\mu^{2 / h_{2}} e^{\left(a_{3}\left(l_{2}+1\right)-a_{2}\left(l_{2}+3\right)-a_{1}\left(l_{2}-2\right)\right) / l_{2}}$. If $0<\varepsilon<1$ is satisfied, then one obtains $\lambda=-(1 / 2) \ln (\varepsilon)$. Integrating all cases (A.9)-(A.13), we have $V_{\sigma(t)}(t)<e^{-2 \lambda t} V_{1}(0)$, which means that system (8) is exponentially stable for system (8) with $\omega(t) \equiv 0$, where $\lambda$ and $\beta$ are given in Proposition 2 .

Now, we are in a position to show the $H_{\infty}$ performance for system (8) with nonzero $\omega(t) \in \mathscr{L}_{2}[0, \infty)$ under the zero initial condition.

Using Lemma 1 and Jensen's inequality to (A.2), we have

$$
\begin{gathered}
\dot{V}_{1}(t)+z^{T}(t) z(t)-\gamma^{2} \omega^{T}(t) \omega(t) \\
\leq-a_{1} V_{1}(t)+\xi_{1}^{T}(t) \Xi^{(1)} \xi_{1}(t),
\end{gathered}
$$

where $\xi_{1}^{T}(t)=\left[x^{T}(t) x^{T}\left(t-\tau_{1}(t)\right) x^{T}\left(t-h_{1}\right) x^{T}(t-\right.$ $\left.\left.h_{2}\right) \omega^{T}(t)\right]$.

Similarly, we obtain

$$
\begin{gathered}
\dot{V}_{2}(t)+z^{T}(t) z(t)-\gamma^{2} \omega^{T}(t) \omega(t) \\
\leq-a_{2} V_{2}(t)+\xi_{i 1}^{T}(t) \Xi^{(i 1)} \xi_{i 1}(t), \\
\dot{V}_{3}(t)+z^{T}(t) z(t)-\gamma^{2} \omega^{T}(t) \omega(t) \\
\leq-a_{3} V_{3}(t)+\xi_{i 2}^{T}(t) \Xi^{(i 2)} \xi_{i 2}(t),
\end{gathered}
$$

where $\xi_{i j}^{T}(t)=\left[x^{T}(t) \quad x^{T}\left(t-\tau_{i}^{(1)}(t)\right) \quad x^{T}\left(t-\tau_{i}^{(2)}(t)\right) \quad x^{T}(t-\right.$ $\left.\left.h_{1}\right) x^{T}\left(t-h_{2}\right) \omega^{T}(t)\right](j=1,2)$.

It follows from (20)-(22) and (A.14)-(A.15) that

$$
\begin{aligned}
& \dot{V}_{\sigma(t)}(t)+z^{T}(t) z(t)-\gamma^{2} \omega^{T}(t) \omega(t) \\
& \quad \leq-a_{\sigma(t)} V_{\sigma(t)}(t) .
\end{aligned}
$$

Let $\mathscr{I}_{i}=\left[t_{i}, t_{i+1}\right)$. When $t \in \mathscr{I}_{i}$, integrating both sides of (A.16) from $t_{i}$ to $t$, we have

$$
\begin{aligned}
V_{\sigma\left(t_{i}^{+}\right)}(t) \leq & e^{-a_{\sigma\left(t_{i}^{+}\right)}\left(t-t_{i}\right)} V_{\sigma\left(t_{i}^{+}\right)}\left(t_{i}^{+}\right) \\
& -\int_{t_{i}}^{t} e^{-a_{\sigma\left(t_{i}^{+}\right)}(t-s)} \Delta(s) d s,
\end{aligned}
$$

where $\Delta(s)=z^{T}(s) z(s)-\gamma^{2} \omega^{T}(s) \omega(s)$.

Combining (A.6) and (A.17), we obtain

$$
\begin{aligned}
V_{\sigma\left(t_{i}^{+}\right)}(t) \leq & e^{-a_{\sigma\left(t_{i}^{+}\right)}\left(t-t_{i}\right)} V_{\sigma\left(t_{i}^{+}\right)}\left(t_{i}^{+}\right) \\
& -\int_{t_{i}}^{t} e^{-a_{\sigma\left(t_{i}^{+}\right)}(t-s)} \Delta(s) d s \\
\leq & \widehat{v} e^{-a_{\sigma\left(t_{i}^{+}\right)}\left(t-t_{i}\right)} V_{\sigma\left(t_{i}^{-}\right)}\left(t_{i}^{-}\right) \\
& -\int_{t_{i}}^{t} e^{-a_{\sigma\left(t_{i}^{+}\right)}(t-s)} \Delta(s) d s \leq \cdots \\
\leq & e^{-2 \lambda t} V_{1}(0)-\int_{0}^{t} e^{-2 \lambda(t-s)} \Delta(s) d s,
\end{aligned}
$$

where

$\widehat{v}$

$$
= \begin{cases}v_{1}, & t_{i}=k l_{1} h_{1}, \\ \mu, & t_{i}=k l_{1} h_{1}+h_{1}, k l_{1} h_{1}+j h_{2}, j=1,2, \ldots, l_{2}-1, \\ v_{2}, & t_{i}=k l_{1} h_{1}+\left(m_{j}+1\right) h_{1} .\end{cases}
$$

For $t \in[0, \infty)$, it can be seen that

$$
V_{\sigma(t)}(t) \leq e^{-2 \lambda t} V_{1}(0)-\int_{0}^{t} e^{-2 \lambda(t-s)} \Delta(s) d s .
$$


Multiplying both sides of (A.20) by $\mu^{-2 t / h_{2}}$ yields

$$
\begin{gathered}
\mu^{-2 t / h_{2}} V_{\sigma(t)}(t)+\int_{0}^{t} e^{-2 \lambda(t-s)-\left(2 t / h_{2}\right) \ln \mu} z^{T}(s) z(s) d s \\
\leq e^{-2 \lambda t-\left(2 t / h_{2}\right) \ln \mu} V_{1}(0) \\
\quad+\int_{0}^{t} e^{-2 \lambda(t-s)-\left(2 t / h_{2}\right) \ln \mu} \gamma^{2} \omega^{T}(s) \omega(s) d s \\
\leq e^{-2 \lambda t-\left(2 t / h_{2}\right) \ln \mu} V_{1}(0)+\int_{0}^{t} \gamma^{2} \omega^{T}(s) \omega(s) d s .
\end{gathered}
$$

Taking zero initial condition and $V_{\sigma(t)}(t)>0$ into consideration, we have

$$
\begin{gathered}
\int_{0}^{t} e^{-2 \lambda(t-s)-\left(2 t / h_{2}\right) \ln \mu} z^{T}(s) z(s) d s \\
<\int_{0}^{t} \gamma^{2} \omega^{T}(s) \omega(s) d s .
\end{gathered}
$$

Notice that $-2 \lambda(t-s)-\left(2 t / h_{2}\right) \ln \mu=-\alpha t+2 \lambda s>-\alpha t$, where $\alpha=\left(a_{1}\left(l_{2}-2\right)+a_{2}\left(l_{2}+3\right)-a_{3}\left(l_{2}+1\right)\right) / l_{2}$. So it is easy to obtain

$$
\int_{0}^{t} e^{-\alpha t} z^{T}(s) z(s) d s \leq \gamma^{2} \int_{0}^{t} \omega^{T}(s) \omega(s) d s .
$$

Integrating both sides of (A.23) from $t=0$ to $t=\infty$ leads to

$$
\int_{0}^{\infty} e^{-\alpha s} z^{T}(s) z(s) d s \leq \gamma^{2} \int_{0}^{\infty} \omega^{T}(s) \omega(s) d s
$$

This completes the proof.

\section{B. Proof of Proposition 3}

Proof. By Schur complement, (20) is equivalent to

$$
\widehat{\Xi}^{(1)}=\left[\begin{array}{cc}
\widehat{\Pi}_{11}^{(1)} & \widehat{\Pi}_{12}^{(1)} \\
* & \widehat{\Pi}_{22}^{(1)}
\end{array}\right]<0
$$

where

$$
\widehat{\Pi}_{11}^{(1)}=\left[\begin{array}{ccccc}
\widehat{\Xi}_{11}^{(1)} & \Xi_{12}^{(1)} & 0 & 0 & P_{1} E+C^{T} D \\
* & \Xi_{22}^{(1)} & \Xi_{23}^{(1)} & 0 & 0 \\
* & * & \Xi_{33}^{(1)} & \Xi_{34}^{(1)} & 0 \\
* & * & * & \Xi_{44}^{(1)} & 0 \\
* & * & * & * & -\gamma^{2} I+D^{T} D
\end{array}\right],
$$

$$
\begin{aligned}
\widehat{\Pi}_{12}^{(1)} & =\left[\begin{array}{ccc}
h_{1} A^{T} & h_{12} A^{T} & C^{T} \\
h_{1} F_{1}^{T} B^{T} & h_{12} F_{1}^{T} B^{T} & 0 \\
0 & 0 & 0 \\
0 & 0 & 0 \\
h_{1} E^{T} & h_{12} E^{T} & 0
\end{array}\right], \\
\widehat{\Pi}_{22}^{(1)} & =\left[\begin{array}{ccc}
R_{11}^{-1} & 0 & 0 \\
* & R_{21}^{-1} & 0 \\
* & * & -I
\end{array}\right], \\
\widehat{\Xi}_{11}^{(1)} & =A^{T} P_{1}+P_{1} A+a_{1} P_{1}+Q_{11}-\sigma_{11} R_{11} .
\end{aligned}
$$

Define $\Delta_{1}=\operatorname{diag}\left\{P_{1}^{-1}, P_{1}^{-1}, P_{1}^{-1}, P_{1}^{-1}, I, I, I, I\right\}$. Performing a congruence transformation to (B.1) by $\Delta_{1}$, together with the changes of matrix variables defined by $\bar{P}_{1}=P_{1}^{-1}, \bar{Q}_{i 1}=$ $\bar{P}_{1} Q_{i 1} \bar{P}_{1}(i=1,2), \bar{R}_{i 1}=\bar{P}_{1} R_{i 1} \bar{P}_{1}(i=1,2), \bar{S}_{1}=\bar{P}_{1} S_{1} \bar{P}_{1}$, $\bar{F}_{1}=F_{1} \bar{P}_{1}$, and using $-\bar{P}_{1} \bar{R}_{i 1}^{-1} \bar{P}_{1} \leq v^{2} \bar{R}_{i 1}-2 v \bar{P}_{1}(i=1,2)$, we can obtain (30).

Using a similar method to (21) and (22), we can obtain (31) and (32), respectively, where $\bar{P}_{j}=P_{j}^{-1}, \bar{S}_{2}=\bar{P}_{2} S_{2} \bar{P}_{2}$, $\bar{Q}_{i j}=\bar{P}_{j} Q_{i j} \bar{P}_{j}, \bar{R}_{i j}=\bar{P}_{j} R_{i j} \bar{P}_{j}, \bar{S}_{2,1}=\bar{P}_{2} S_{2,1} \bar{P}_{2}, \bar{S}_{2,2}=\bar{P}_{2} S_{2,2} \bar{P}_{2}$, $\bar{S}_{3,1}=\bar{P}_{3} S_{3,1} \bar{P}_{3}, \bar{S}_{3,2}=\bar{P}_{3} S_{3,2} \bar{P}_{3}, \bar{F}_{1 j}=F_{j} I_{p_{1}} \bar{P}_{j}$, and $\bar{F}_{2 j}=$ $F_{j} I_{p_{2}} \bar{P}_{j}(i=1,2 ; j=1,2,3)$. Before and after multiplying the inequalities $P_{1} \leq \mu P_{2}, P_{2} \leq \mu P_{1}, P_{3} \leq \mu P_{2}$, and $P_{2} \leq \mu P_{3}$ by $P_{2}^{-1}, P_{1}^{-1}, P_{2}^{-1}$, and $P_{3}^{-1}$, respectively, we have $\bar{P}_{2} \leq \mu \bar{P}_{1}$, $\bar{P}_{1} \leq \mu \bar{P}_{2}, \bar{P}_{2} \leq \mu \bar{P}_{3}$, and $\bar{P}_{3} \leq \mu \bar{P}_{2}$. Similarly, we obtain $\bar{Q}_{i 2} \leq \mu \bar{Q}_{i 1}, \bar{Q}_{i 1} \leq \mu \bar{Q}_{i 2}, \bar{Q}_{i 2} \leq \mu \bar{Q}_{i 3}, \bar{Q}_{i 3} \leq \mu \bar{Q}_{i 2}, \bar{R}_{i 2} \leq$ $\mu \bar{R}_{i 1}, \bar{R}_{i 1} \leq \mu \bar{R}_{i 2}, \bar{R}_{i 2} \leq \mu \bar{R}_{i 3}, \bar{R}_{i 3} \leq \mu \bar{R}_{i 2}(i=1,2)$, and the equalities (26)-(29). The proof is completed.

\section{Conflict of Interests}

The authors declare that there is no conflict of interests regarding the publication of this paper.

\section{Acknowledgments}

This work was supported in part by the Natural Science Foundation of China under Grants 61403240, 61374059, and U1334210, Scientific and Technologial Innovation Programs of Higher Education Institutions in Shanxi under Grant 2013105, International S\&T Cooperation Program of Shanxi Province, China, under Grant 2013081040.

\section{References}

[1] T. Chen and F. Bruce, Optimal Sampled-Data Control Systems, Springer, London, UK, 1995.

[2] Z. Sun, "Sampling and control of switched linear systems," Journal of the Franklin Institute, vol. 341, no. 7, pp. 657-674, 2004.

[3] L. Qiu and T. Chen, "Multirate sampled-data systems: all $H_{\infty}$ suboptimal controllers and the minimum entropy controller," 
IEEE Transactions on Automatic Control, vol. 44, no. 3, pp. 537550, 1999.

[4] T. Chen and L. Qiu, " $H_{\infty}$ design of general multirate sampleddata control systems," Automatica, vol. 30, no. 7, pp. 1139-1152, 1994.

[5] T. Chen, "On stability robustness of a dual-rate control system," IEEE Transactions on Automatic Control, vol. 39, no. 1, pp. 164$167,1994$.

[6] J. Ding, F. Ding, X. P. Liu, and G. Liu, "Hierarchical least squares identification for linear SISO systems with dual-rate sampleddata," IEEE Transactions on Automatic Control, vol. 56, no. 11, pp. 2677-2683, 2011.

[7] J.-S. Yang and R. G. Pietila Jr., "Parameter plane control design for a stirred-tank chemical system with slow-fast multirate sampling," Journal of the Franklin Institute, vol. 330, no. 6, pp. 1177-1193, 1993.

[8] D. P. Glasson, "Development and applications of multi-rate digital control," IEEE Control Systems Magazine, vol. 3, no. 4, pp. 2-8, 1983.

[9] M. Mizuochi, T. Tsuji, and K. Ohnishi, "Multirate sampling method for acceleration control system," IEEE Transactions on Industrial Electronics, vol. 54, no. 3, pp. 1462-1471, 2007.

[10] R. D. Gudi, S. L. Shah, and M. R. Gray, "Multirate state and-parameter estimation in an antibiotic fermentation with delayed measurements," Biotechnology and Bioengineering, vol. 44, no. 11, pp. 1271-1278, 1994.

[11] S. Hutchinson, G. D. Hager, and P. I. Corke, "A tutorial on visual servo control," IEEE Transactions on Robotics and Automation, vol. 12, no. 5, pp. 651-670, 1996.

[12] M. Nemani, T.-C. Tsao, and S. Hutchinson, "Multi-rate analysis and design of visual feedback digital servo-control system," Journal of Dynamic Systems, Measurement and Control, vol. 116, no. 1, pp. 45-55, 1994.

[13] M. Cimino and P. R. Pagilla, "A design technique for multirate linear systems," IEEE Transactions on Control Systems Technology, vol. 17, no. 6, pp. 1342-1349, 2009.

[14] S.-H. Lee, "Multirate digital control system design and its application to computer disk drives," IEEE Transactions on Control Systems Technology, vol. 14, no. 1, pp. 124-133, 2006.

[15] J. Wang, T. Chen, and B. Huang, "Multirate sampled-data systems: computing fast-rate models," Journal of Process Control, vol. 14, no. 1, pp. 79-88, 2004.

[16] E. Fridman, A. Seuret, and J.-P. Richard, "Robust sampleddata stabilization of linear systems: an input delay approach," Automatica, vol. 40, no. 8, pp. 1441-1446, 2004.

[17] P. Naghshtabrizi, J. P. Hespanha, and A. R. Teel, "Exponential stability of impulsive systems with application to uncertain sampled-data systems," Systems and Control Letters, vol. 57, no. 5, pp. 378-385, 2008.

[18] H. Shao and Q.-L. Han, "On stabilization for systems with two additive time-varying input delays arising from networked control systems," Journal of the Franklin Institute, vol. 349, no. 6, pp. 2033-2046, 2012.

[19] D. Yue, Q.-L. Han, and J. Lam, "Network-based robust $H_{\infty}$ control of systems with uncertainty," Automatica, vol. 41, no. 6 , pp. 999-1007, 2005.

[20] K. Liu, E. Fridman, and L. Hetel, "Stability and $L_{2}$-gain analysis of networked control systems under Round-Robin scheduling: a time-delay approach," Systems \& Control Letters, vol. 61, no. 5, pp. 666-675, 2012.
[21] K. Liu and E. Fridman, "Wirtinger's inequality and Lyapunovbased sampled-data stabilization," Automatica, vol. 48, no. 1, pp. 102-108, 2012.

[22] K. Liu and E. Fridman, "Networked-based stabilization via discontinuous Lyapunov functionals," International Journal of Robust and Nonlinear Control, vol. 22, no. 4, pp. 420-436, 2012.

[23] D. Zhang, Q.-L. Han, and X. Jia, “Observer-based $H_{\infty}$ output tracking control for networked control systems," International Journal of Robust and Nonlinear Control, vol. 24, no. 17, pp. 27412760, 2014.

[24] D. Zhang, Q.-L. Han, and X. Jia, "Network-based output tracking control for a class of T-S fuzzy systems that can not be stabilized by nondelayed output feedback controllers," IEEE Transactions on Cybernetics, 2014.

[25] M. Moarref and L. Rodrigues, "Stability and stabilization of linear sampled-data systems with multi-rate samplers and time driven zero order holds," Automatica, vol. 50, no. 10, pp. 26852691, 2014.

[26] S. Zhu, M. Meng, and C. Zhang, "Exponential stability for positive systems with bounded time-varying delays and static output feedback stabilization," Journal of the Franklin Institute. Engineering and Applied Mathematics, vol. 350, no. 3, pp. 617636, 2013.

[27] C. Peng and M.-R. Fei, "An improved result on the stability of uncertain T-S fuzzy systems with interval time-varying delay," Fuzzy Sets and Systems, vol. 212, pp. 97-109, 2013.

[28] X. Jia, L. Li, D. Zhang, X. Chi, and X. Fan, "Exponential stabilization of dual-rate control system: a switched system approach," in Proceedings of the 1st American Control Conference (ACC '13), pp. 48-53, Washington, DC, USA, June 2013.

[29] W. Zhiwen and G. Ge, "Fundamental issues and prospective directions in networked multirate control systems," Mathematical Problems in Engineering, vol. 2014, Article ID 513506, 10 pages, 2014.

[30] C. C. MacAdam, "Application of an optimal preview control for simulation of closed-loop automobile driving," IEEE Transactions on Systems, Man and Cybernetics, vol. 11, no. 6, pp. 393399, 1981. 


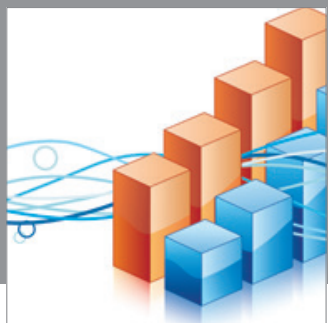

Advances in

Operations Research

mansans

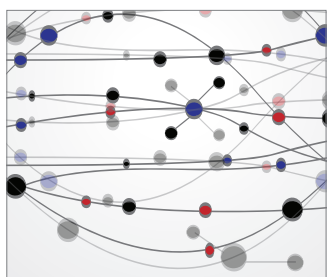

The Scientific World Journal
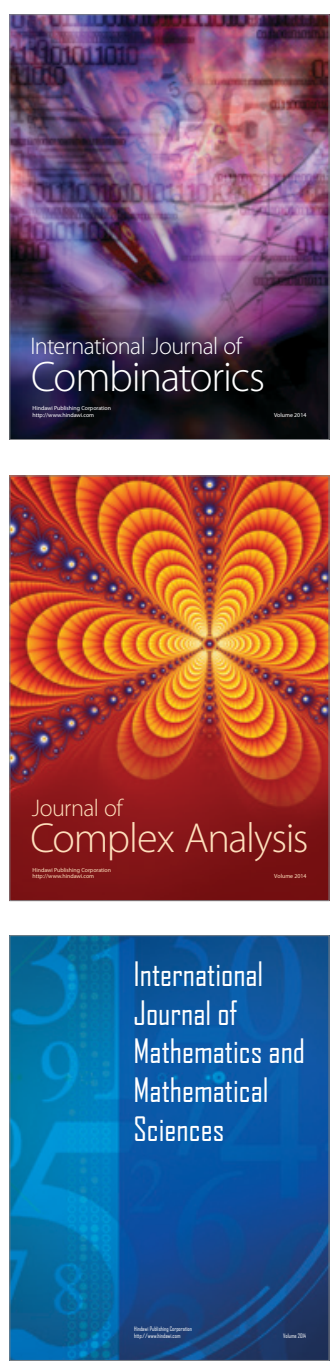
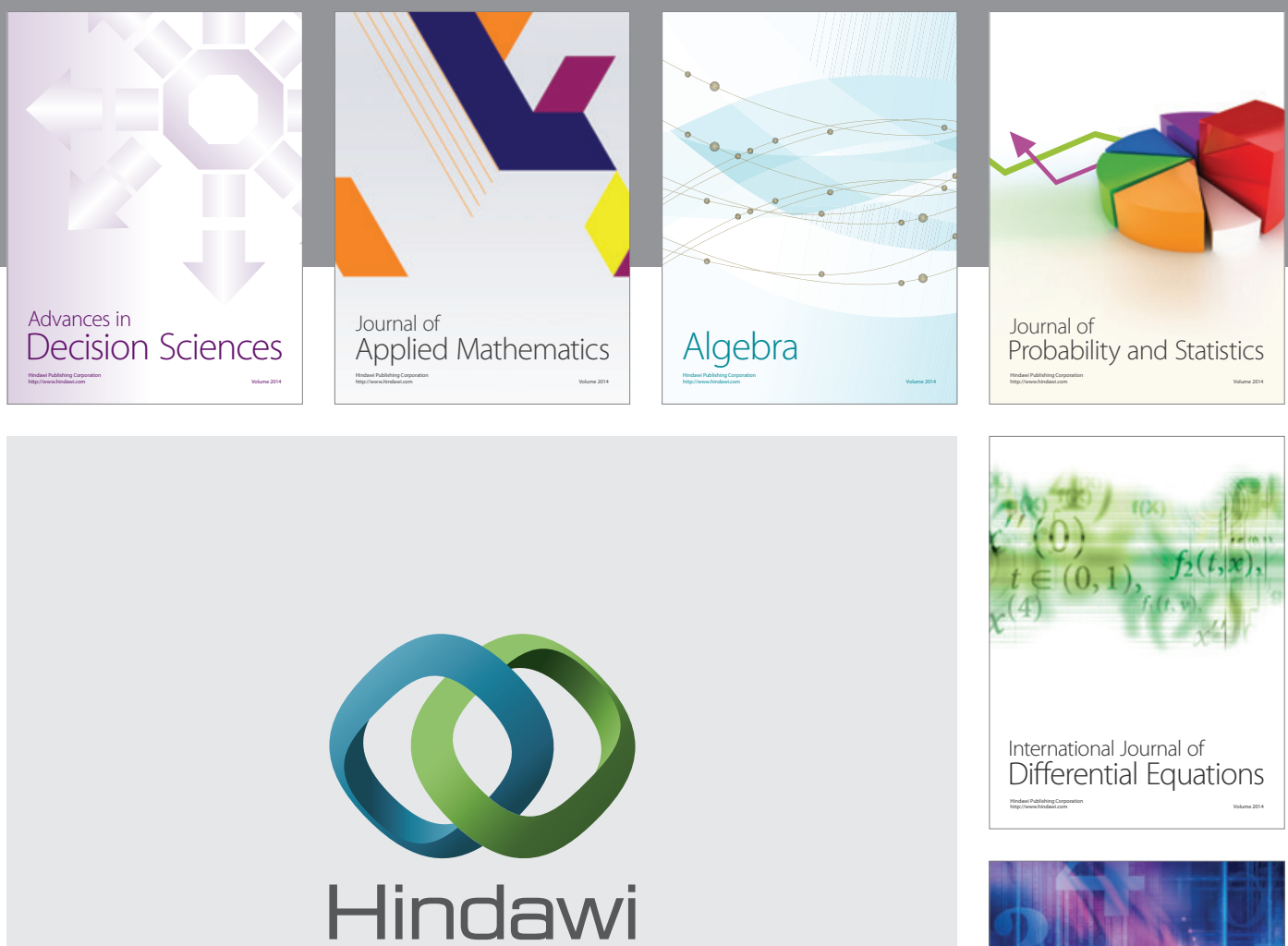

Submit your manuscripts at http://www.hindawi.com
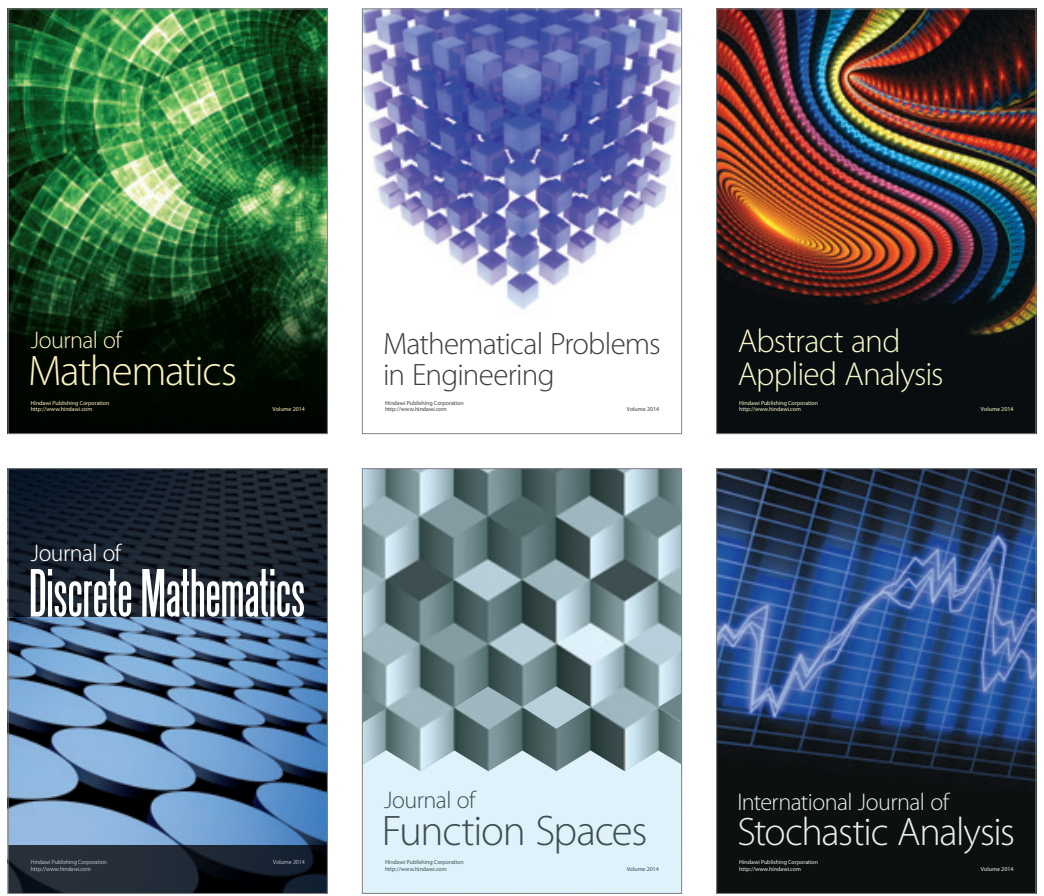

Journal of

Function Spaces

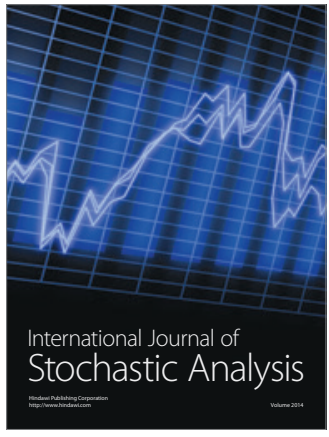

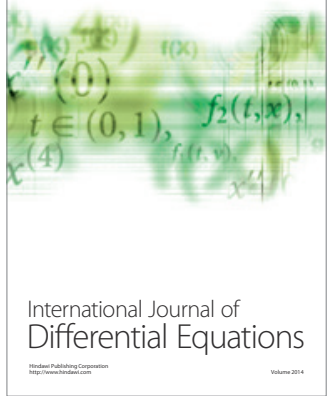
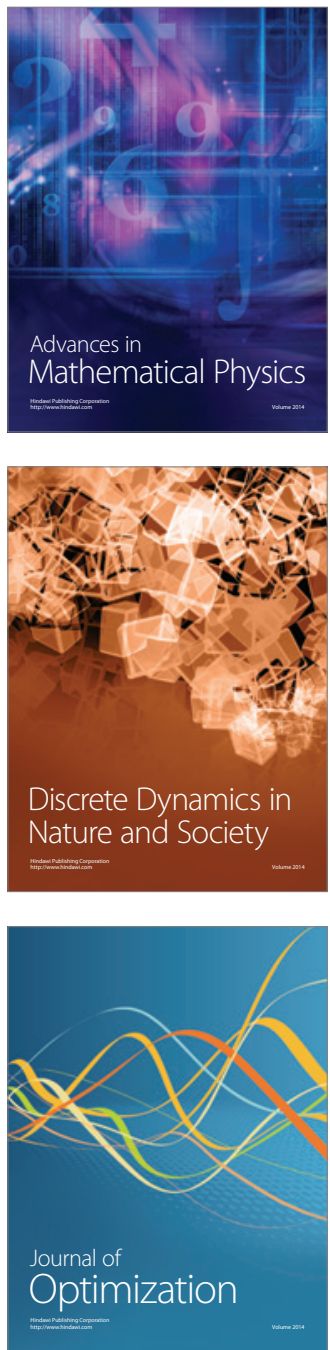